\title{
Stellar ages, masses, and radii from asteroseismic modeling are robust to systematic errors in spectroscopy
}

\author{
E. P. Bellinger ${ }^{1, \star}$, S. Hekker ${ }^{2,1}$, G. C. Angelou ${ }^{3}$, A. Stokholm ${ }^{1}$, and S. Basu ${ }^{4}$ \\ 1 Stellar Astrophysics Centre, Department of Physics and Astronomy, Aarhus University, Denmark \\ e-mail: bellinger@phys .au.dk \\ 2 Stellar Ages \& Galactic Evolution (SAGE) Group, Max Planck Institute for Solar System Research, Göttingen, Germany \\ 3 Max Planck Institute for Astrophysics, Garching, Germany \\ ${ }^{4}$ Department of Astronomy, Yale University, New Haven, CT, USA
}

Received 19 October 2018 / Accepted 13 December 2018

\begin{abstract}
Context. The search for twins of the Sun and Earth relies on accurate characterization of stellar and the exoplanetary parameters age, mass, and radius. In the modern era of asteroseismology, parameters of solar-like stars are derived by fitting theoretical models to observational data, which include measurements of their oscillation frequencies, metallicity $[\mathrm{Fe} / \mathrm{H}]$, and effective temperature $T_{\text {eff }}$. Furthermore, combining this information with transit data yields the corresponding parameters for their associated exoplanets.

Aims. While values of $[\mathrm{Fe} / \mathrm{H}]$ and $T_{\text {eff }}$ are commonly stated to a precision of $\sim 0.1$ dex and $\sim 100 \mathrm{~K}$, the impact of systematic errors in their measurement has not been studied in practice within the context of the parameters derived from them. Here we seek to quantify this.

Methods. We used the Stellar Parameters in an Instant (SPI) pipeline to estimate the parameters of nearly 100 stars observed by Kepler and Gaia, many of which are confirmed planet hosts. We adjusted the reported spectroscopic measurements of these stars by introducing faux systematic errors and, separately, artificially increasing the reported uncertainties of the measurements, and quantified the differences in the resulting parameters.

Results. We find that a systematic error of $0.1 \mathrm{dex}$ in $[\mathrm{Fe} / \mathrm{H}]$ translates to differences of only $4 \%, 2 \%$, and $1 \%$ on average in the resulting stellar ages, masses, and radii, which are well within their uncertainties $(\sim 11 \%, 3.5 \%, 1.4 \%)$ as derived by SPI. We also find that increasing the uncertainty of $[\mathrm{Fe} / \mathrm{H}]$ measurements by 0.1 dex increases the uncertainties of the ages, masses, and radii by only $0.01 \mathrm{Gyr}, 0.02 M_{\odot}$, and $0.01 R_{\odot}$, which are again well below their reported uncertainties $\left(\sim 0.5 \mathrm{Gyr}, 0.04 M_{\odot}, 0.02 R_{\odot}\right)$. The results for $T_{\text {eff }}$ at $100 \mathrm{~K}$ are similar.

Conclusions. Stellar parameters from SPI are unchanged within uncertainties by errors of up to 0.14 dex or $175 \mathrm{~K}$. They are even more robust to errors in $T_{\text {eff }}$ than the seismic scaling relations. Consequently, the parameters for their exoplanets are also robust.
\end{abstract}

Key words. asteroseismology - stars: abundances - stars: low-mass - planets and satellites: fundamental parameters stars: oscillations - stars: evolution

\section{Introduction}

The modern study of cool dwarf stars has been revolutionized in recent years by ultraprecise measurements of low-amplitude global stellar pulsations (for a summary of early results, see Chaplin \& Miglio 2013). Traveling through the star before emerging near the surface, pulsations bring information to light about the otherwise opaque conditions of the stellar interior, and thereby provide numerous constraints to the internal structure and composition of stars. Indeed, connecting theoretical stellar models to measurements of pulsation frequencies - as well as other measurements, such as those derived from spectroscopy yields precise determinations of stellar parameters such as age, mass, and radius (for an overview, see Basu \& Chaplin 2017).

Precisely constrained stellar parameters are broadly useful for a variety of endeavours, such as testing theories of stellar and galactic evolution (e.g., Deheuvels et al. 2016; Hjørringgaard et al. 2017; Nissen et al. 2017; Bellinger et al. 2017) and mapping out history and dynamics of the Galaxy (e.g., Chiappini et al. 2015;

^ SAC Postdoctoral Fellow.
Anders et al. 2017; Silva Aguirre et al. 2018; Feuillet et al. 2018). Combining stellar parameters with transit measurements furthermore yields properties of the exoplanets that they harbor, such as their masses, radii, obliquities, eccentricities, and semi-major axes (e.g., Seager \& Mallén-Ornelas 2003; Huber et al. 2013; Van Eylen et al. 2014; Huber 2016, 2018; Campante et al. 2016; Kamiaka et al. 2018; Adibekyan et al. 2018). This then permits tests to theories of planet formation (e.g., Lin et al. 1996; Watson et al. 2011; Morton \& Johnson 2011; Lai et al. 2011; Guillochon et al. 2011; Thies et al. 2011; Teyssandier et al. 2013; Plavchan \& Bilinski 2013; Matsakos \& Königl 2015, 2017; Li \& Winn 2016; Königl et al. 2017; Gratia \& Fabrycky 2017) and facilitates the search for habitable exoplanets.

From first principles, one may obtain approximate relations to deduce stellar radii $(R)$, mean densities $(\rho)$, and masses $(M)$ from asteroseismic data by scaling the values of the Sun to the observed properties of other stars ("scaling relations," Ulrich 1986; Brown et al. 1991; Kjeldsen \& Bedding 1995):

$$
\frac{R}{R_{\odot}} \simeq\left(\frac{v_{\max }}{v_{\max , \odot}}\right)\left(\frac{\Delta v}{\Delta v_{\odot}}\right)^{-2}\left(\frac{T_{\mathrm{eff}}}{T_{\mathrm{eff}, \odot}}\right)^{\frac{1}{2}}
$$


$\frac{\rho}{\rho_{\odot}} \simeq\left(\frac{\Delta v}{\Delta v_{\odot}}\right)^{2}\left(\frac{T_{\mathrm{eff}}}{T_{\mathrm{eff}, \odot}}\right)$

$\frac{M}{M_{\odot}} \simeq\left(\frac{v_{\max }}{v_{\max , \odot}}\right)^{3}\left(\frac{\Delta v}{\Delta v_{\odot}}\right)^{-4}\left(\frac{T_{\mathrm{eff}}}{T_{\mathrm{eff}, \odot}}\right)^{\frac{3}{2}}$

Here $T_{\text {eff }}$ is the effective temperature of the star, $v_{\max }$ is the frequency of maximum oscillation power, and $\Delta v$ is the large frequency separation (for detailed definitions, see, e.g., Basu \& Chaplin 2017). The quantities subscripted with the solar symbol $(\odot)$ correspond to the solar values: $v_{\max , \odot}=3090 \pm 30 \mu \mathrm{Hz}$, $\Delta v_{\odot}=135.1 \pm 0.1 \mu \mathrm{Hz}$, and $T_{\text {eff, } \odot}=5772.0 \pm 0.8 \mathrm{~K}$ (Huber et al. 2011; Prša et al. 2016). This way of estimating stellar parameters is often referred to as the "direct method" (e.g., Lundkvist et al. 2018) because it is independent of stellar models and relies only on physical arguments. We note that there are no such scaling relations for the stellar age.

The seismic scaling relations are all functions of the stellar effective temperature, which, along with metallicity, can be derived from spectroscopic observations of a star. A variety of factors contribute to the determination of these spectroscopic parameters, such as spectra normalization, corrections for pixelto-pixel variations, the placement of the continuum, and so on (e.g., Massey \& Hanson 2013; Škoda 2017). The analysis can be complicated by effects such as line blending and broadening. Uncertainties in atmospheric models and the techniques for obtaining atmospheric parameters such as the measurement of equivalent widths, fitting on synthetic spectra, and degeneracies between spectroscopic parameters can also cause difficulties. Often, parameters for targets in large spectroscopic surveys are extracted automatically without visual inspection, which can lead to poor fits that sometimes go unnoticed. Conversely, analysis based on visual inspection sometimes leads to different practitioners coming to different conclusions about the same star. Thus there are opportunities for systematic errors to be introduced into the determined effective temperatures and metallicities (e.g., Creevey et al. 2013). However, to date, no study has systematically tested in practice the impact of systematic errors in spectroscopic parameters on the determination of stellar parameters for a large sample of stars.

Unlike the effective temperature, the classical scaling relations presented above are not functions of metallicity, and so one would expect metallicity to have little importance in the determination of stellar masses and radii. However, these relations are approximations, and are not perfectly accurate. Several studies (e.g., White et al. 2011; Belkacem et al. 2011; Mathur et al. 2012; Hekker et al. 2013; Mosser et al. 2013; Huber et al. 2014, 2017; Guggenberger et al. 2016, 2017; Sharma et al. 2016; Gaulme et al. 2016; Viani et al. 2017; Themeß1 et al. 2018) have sought to quantify the validity of the seismic scaling relations especially as a function of stellar evolution, when the assumption of solar homology breaks down - for example by comparison with stellar models, or via orbital analyses of binary stars. Viani et al. (2017) have also recently shown theoretically that these relations neglect a term for the mean molecular weight, which implies a previously unaccounted for dependence on metallicity.

To obtain more accurate mass and radius estimates - and stellar ages - one can fit theoretical models to the observations of a star (e.g., Brown et al. 1994; Gai et al. 2011; Metcalfe et al. 2014; Lebreton \& Goupil 2014; Silva Aguirre et al. 2015, 2017; Bellinger et al. 2016). The connection between observations and stellar parameters then becomes much more complex, relying on the details of not only the coupled differential equations governing the structure and evolution of stars, but also the choices and implementations regarding the microphysics of the star, such as the opacities and equation of state of stellar plasma. This motivates the need for numerical studies to determine the relationships between the observations of a star and the resulting fundamental parameters (e.g., Brown et al. 1994; Angelou et al. 2017; Valle et al. 2018). Here we examine one important aspect of these relations: namely, the impact of systematic errors and underestimated uncertainties in spectroscopic parameters on the determination of stellar parameters via asteroseismic modeling.

In order to perform this study, we used the Stellar Parameters in an Instant pipeline (SPI, Bellinger et al. 2016), which uses machine learning to rapidly connect observations of stars to theoretical models. The SPI method involves training an ensemble or "forest" of decision tree regressors (Breiman 2001; Geurts et al. 2006; Friedman et al. 2001) to learn the relationships between the observable aspects of stars (such as their oscillations) and the unobserved or unobservable aspects (such as their age). We then fed the observations of a particular star into the learned forest, which outputs the desired predicted quantities. In order to propagate the uncertainties of the observations into each of the predicted quantities, we generated random realizations of noise according to the uncertainties of the observations (including their correlations), and ran these random realizations also through the forest. This yields the posterior distribution for each predicted quantity, from which we may obtain, for example, the mean value and its corresponding uncertainty for quantities such as the stellar age, mass, radius, mean density, and luminosity.

Using this approach, one can simultaneously and independently vary multiple free parameters corresponding to uncertain aspects of stellar models, such as the efficiency of convection and the strength of gravitational settling; and therefore propagate many of the uncertainties of the theoretical models into the resulting stellar parameters. To this end, we generated a large grid of stellar models that have been varied in age as well as seven other parameters controlling the physics of the evolution, which is described in more detail in the next section. Since obtaining the parameters of a star with SPI takes less than a minute (rather than hours or days, as is typical with some other methods) we were then able to test the robustness of the final stellar parameters against injections of various amounts of systematic errors.

The question of how systematic errors in spectroscopy impact stellar parameter determinations has direct bearing on exoplanet research. This is due to the fact that the determination of exoplanetary parameters generally depends very strongly on the ability to constrain the parameters of the host star (see Fig. 1).

Given the radius of the host star from asteroseismology (e.g., using Eq. (1); or using more sophisticated methods, such as SPI), and assuming a uniformly bright stellar disk, the radius of a transiting exoplanet can be found by measuring the transit depth (e.g., Seager \& Mallén-Ornelas 2003). Given the stellar radius and mean density (e.g., Eq. (2)), and assuming a circular orbit, one can compute the semi-major axis of an exoplanet from its orbital period. Combining that information with the stellar mass (e.g., Eq. (3)), one can find the mass of an exoplanet using Kepler's third law. As prevailing theories give that planets form roughly around the same time as the host star, the stellar age that is found is generally then attributed to its companions as well (e.g., Jenkins et al. 2015; Campante et al. 2015; Safonova et al. 2016; Christensen-Dalsgaard et al. 2018). From this information, the composition of an exoplanet can be determined by connecting these properties to theoretical models of planet 


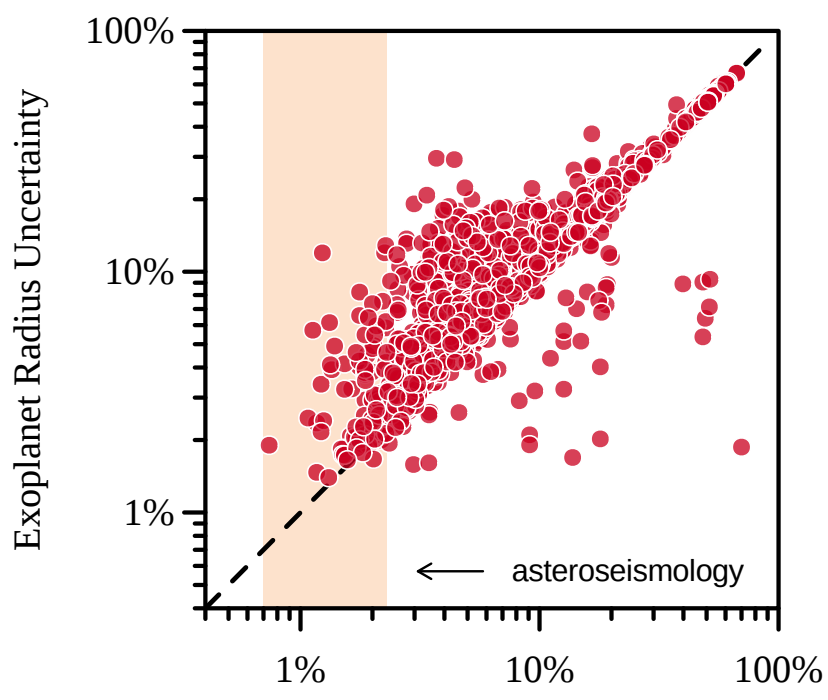

Stellar Radius Uncertainty

Fig. 1. Relative uncertainties of the radii of transiting exoplanets as a function of the relative uncertainty in the radius of their host star. The dashed line shows a one-to-one agreement. The shaded region shows the full range of stellar radii uncertainties determined for the 97 stars examined in this paper. Exoplanet data are taken from the collection by Han et al. (2014)

Table 1. Parameter ranges for the grid of stellar models.

\begin{tabular}{lccc}
\hline \hline Parameter & Symbol & Range & Solar value \\
\hline Mass & $M / M_{\odot}$ & $(0.7,1.8)$ & 1 \\
Mixing length & $\alpha_{\mathrm{MLT}}$ & $(1,3)$ & 1.85 \\
Initial helium & $Y_{0}$ & $(0.22,0.34)$ & 0.273 \\
Initial metallicity & $Z_{0}$ & $(0.0001,0.04)^{a}$ & 0.019 \\
Overshoot & $\alpha_{\mathrm{ov}}$ & $(0.0001,1)^{a}$ & - \\
Undershoot & $\alpha_{\mathrm{us}}$ & $(0.0001,1)^{a}$ & $\leq 0.05^{b}$ \\
Diffusion factor & $D$ & $(0.0001,3)^{a}$ & 1 \\
\hline Eff. temperature & $T_{\mathrm{eff}}$ & $(4000,14000)$ & 5772 \\
Metallicity & {$[\mathrm{Fe} / \mathrm{H}]$} & $(-2.2,0.44)$ & 0 \\
\hline
\end{tabular}

Notes. For reference, values corresponding to a solar-calibrated model and also the derived initial spectroscopic parameter ranges are listed as well. ${ }^{(a)}$ Varied logarithmically. ${ }^{(b)}$ Basu (1997).

structure and formation (e.g., Seager et al. 2007; Weiss \& Marcy 2014; Rogers 2015; Huber 2018).

Thus, if the parameters derived for the host star are biased, then so too will be the parameters for its exoplanets. A differential bias - e.g., a bias that affects mass more than radius - would furthermore impact strongly on matters such as empirical massradius relations (e.g., Seager et al. 2007; Torres et al. 2010).

\section{Methods}

We used Modules for Experiments in Stellar Astrophysics (MESA r10108, Paxton et al. 2011, 2013, 2015, 2018) to construct a grid of stellar models following the procedure given by Bellinger et al. (2016). The initial parameters were varied quasi-randomly in the ranges given in Table 1 . We introduced an additional free parameter, $\alpha_{\mathrm{uv}}$, which controls the efficiency of convective envelope undershooting. As convection is modeled in MESA as a time-dependent diffusive process, under- and over-

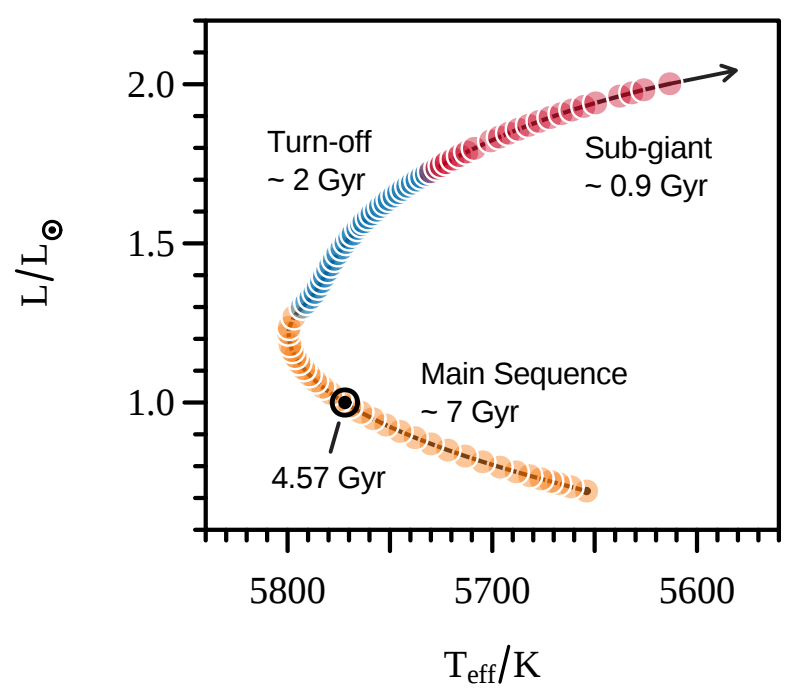

Fig. 2. Theoretical evolution of the Sun in the HR diagram. The position of the present-age Sun is given with the solar symbol $(\odot)$. The points indicate models that have been selected from the track. They are colored according to their assigned phase of evolution, and the approximate duration of each phase is indicated.

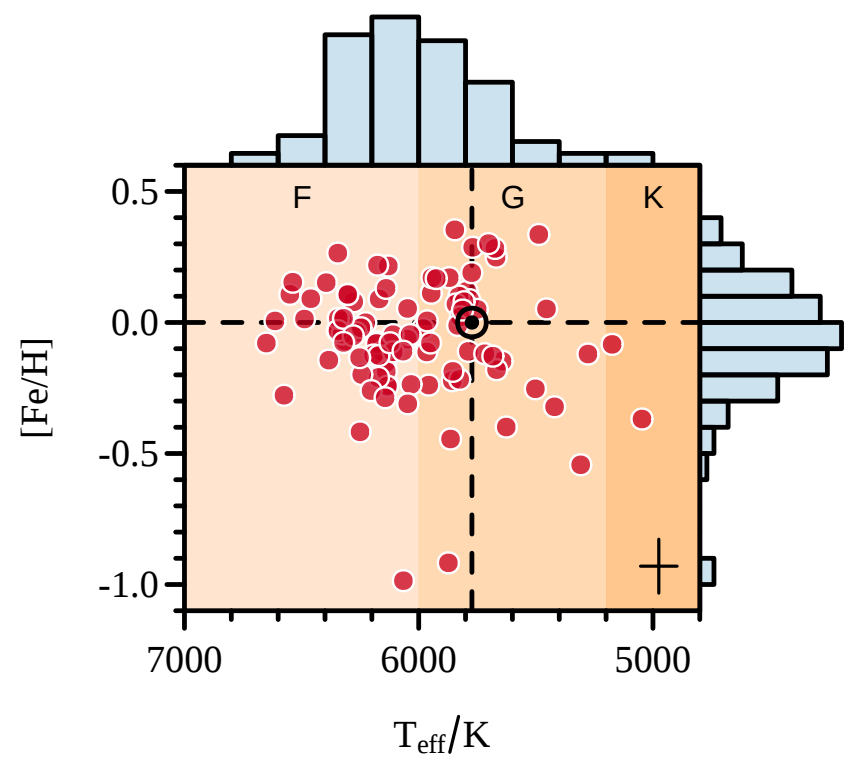

Fig. 3. Spectroscopic measurements of 97 solar-like oscillating stars observed by Kepler. Typical uncertainties $(0.1 \mathrm{dex}, 100 \mathrm{~K})$ are indicated with the cross in the bottom right corner. Histograms are affixed to the top and right side of the figure showing the metallicity and effective temperature distributions for the sample. The values for the Sun are given with the solar symbol $(\odot)$. The background colors indicate the spectral type $(\mathrm{F}, \mathrm{G}, \mathrm{K})$.

shooting are achieved by applying convective velocities to zones within a distance of $\alpha H_{\mathrm{p}}$ beyond the convective boundary, where $\alpha$ is the under- or over-shooting parameter, and $H_{\mathrm{p}}$ is the local pressure scale height. The convective velocities that are used are taken from a distance $f_{0}$ before the boundary; here we used $f_{0}=0.01 H_{\mathrm{p}}$. The remaining aspects of the models are the same as in Bellinger et al. 2016.

We calculated $N=8170$ evolutionary tracks which we simulated from the zero-age main sequence until either an age of $20 \mathrm{Gyr}$ or an asymptotic period spacing of $150 \mathrm{~s}$, which is generally around the base of the red giant branch. Since the 

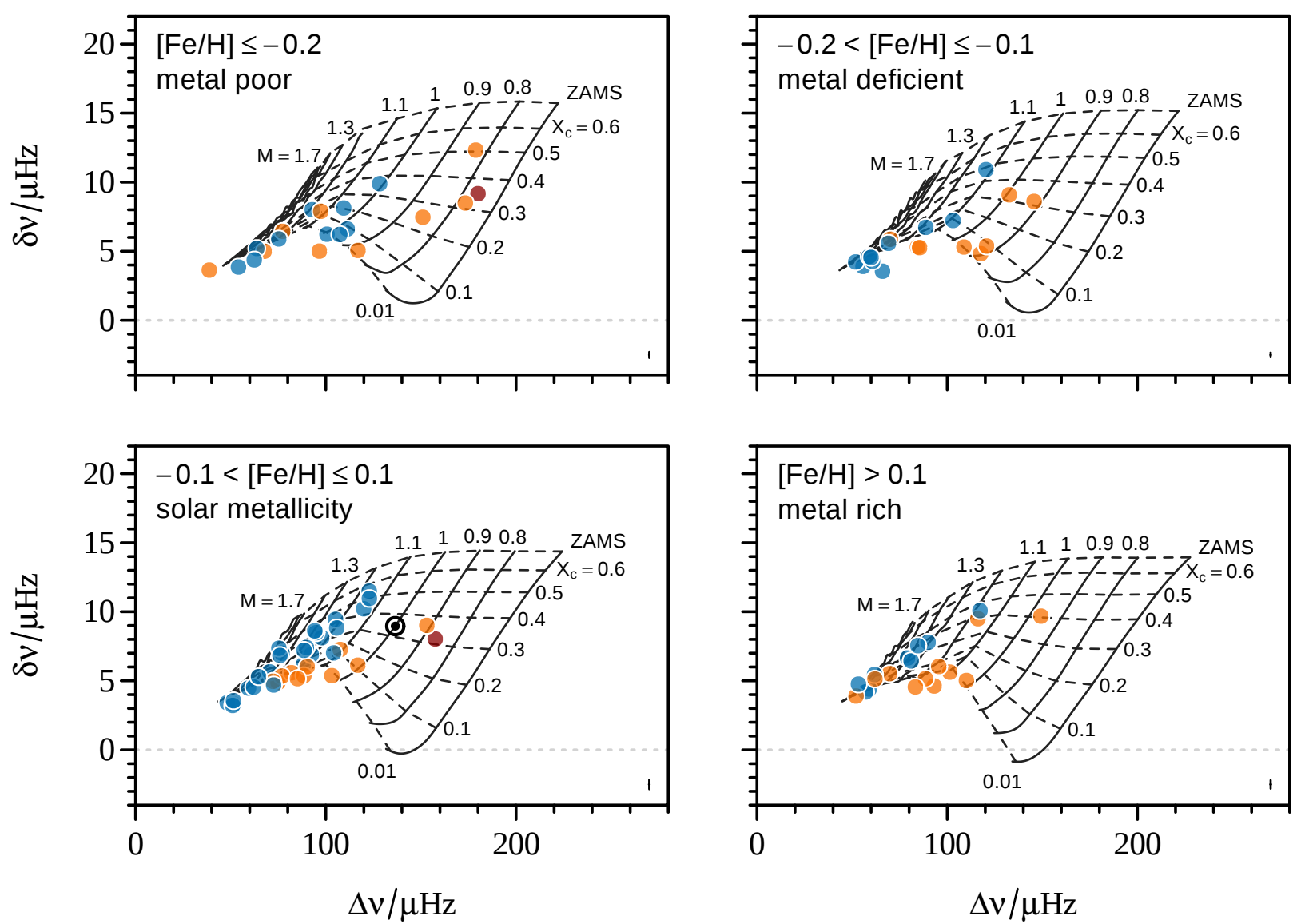

Fig. 4. CD diagram showing asteroseismic measurements - the small frequency separation $\delta v$ and the large frequency separation $\Delta v-$ for the sample of 97 solar-like stars studied here. The panels are arranged by metallicity. The points are colored by spectral type: $\mathrm{F}$ (blue, $T_{\text {eff }}>6000 \mathrm{~K}$ ), $\mathrm{G}$ (orange, $6000 \geq T_{\text {eff }}>5200 \mathrm{~K}$ ), $\mathrm{K}$ (red, $T_{\text {eff }} \leq 5200 \mathrm{~K}$ ). The cross in the bottom right corner of each panel shows typical uncertainties (see also Fig. 5). The dotted light gray line indicates where $\delta v=0$. The solid lines trace theoretical tracks of stellar evolution, with each track labeled along the top by its mass (in solar units) and connected by the dashed lines that are spaced in core-hydrogen abundance $\left(X_{\mathrm{c}}\right)$.

initial conditions of the grid are varied quasi-randomly, the resolution in each parameter $x$ is given by $\left(x_{\max }-x_{\min }\right) / N$; e.g., the typical resolution in mass is approximately $0.0001 M_{\odot}$. From each track we obtained 32 models with core hydrogen abundance $X_{\mathrm{c}}>0.1$ nearly evenly spaced in age (i.e., main-sequence models), 32 models with $0.1 \leq X_{\mathrm{c}} \leq 10^{-6}$ nearly evenly spaced in $\log X_{\mathrm{c}}$ (turn-off models), and 32 models beyond $X_{\mathrm{c}}<10^{-6}$ again nearly evenly spaced in age (sub-giant models). These phases are visualized in Fig. 2.

We calculated $p$-mode frequencies for these models using the GYRE oscillation code (Townsend \& Teitler 2013). We obtained frequency separations and ratios (Roxburgh \& Vorontsov 2003) for these models following the procedures given in Bellinger et al. (2016). We removed models where the presence of mixed modes made it impossible to calculate these quantities.

\section{Data}

We obtained oscillation mode frequencies, $v_{\max }$ values, effective temperatures, and metallicities for 97 stars observed by the Kepler spacecraft (Borucki et al. 2010a) from previous studies. Data for 31 of the stars came from the Kepler Ages project (Silva Aguirre et al. 2015; Davies et al. 2016), and 66 were from the Kepler LEGACY project (Lund et al. 2017; Silva Aguirre et al. 2017). The spectroscopic parameters of these stars are visualized in Fig. 3. We processed the mode frequencies to obtain astero- seismic frequency separations and ratios in the same way that we processed the theoretical model frequencies in the previous section. These stars are visualized in the so-called CD diagram (Christensen-Dalsgaard 1984) in Fig. 4. The relative uncertainties on the observational constraints for these stars are visualized in Fig. 5. This figure furthermore illustrates the observational data we input to SPI.

For comparison purposes, we additionally obtained luminosity and radius measurements for these stars from the recent Gaia Data Release 2 (DR2, Gaia Collaboration 2018; Andrae et al. 2018). We identified these stars using the 2MASS cross-matched catalog (Marrese et al. 2019) for all except KIC 10514430 and KIC 8379927, which we located using a cone search. We note that these measurements have not been corrected for extinction.

\section{Results}

We applied SPI to the sample of 97 stars using the grid of theoretical models that we generated and the observational constraints shown in Fig. 5. Random realizations of the observations that fell outside of the range of the grid of models were dropped. Figure 6 shows the relative uncertainties in the estimated stellar parameters for this sample. The stellar parameters themselves can be found in Table A.1. Figure 7 shows the positions of these stars on the Hertzsprung-Russell diagram using effective temperatures from spectroscopy and predicted luminosities from SPI. 


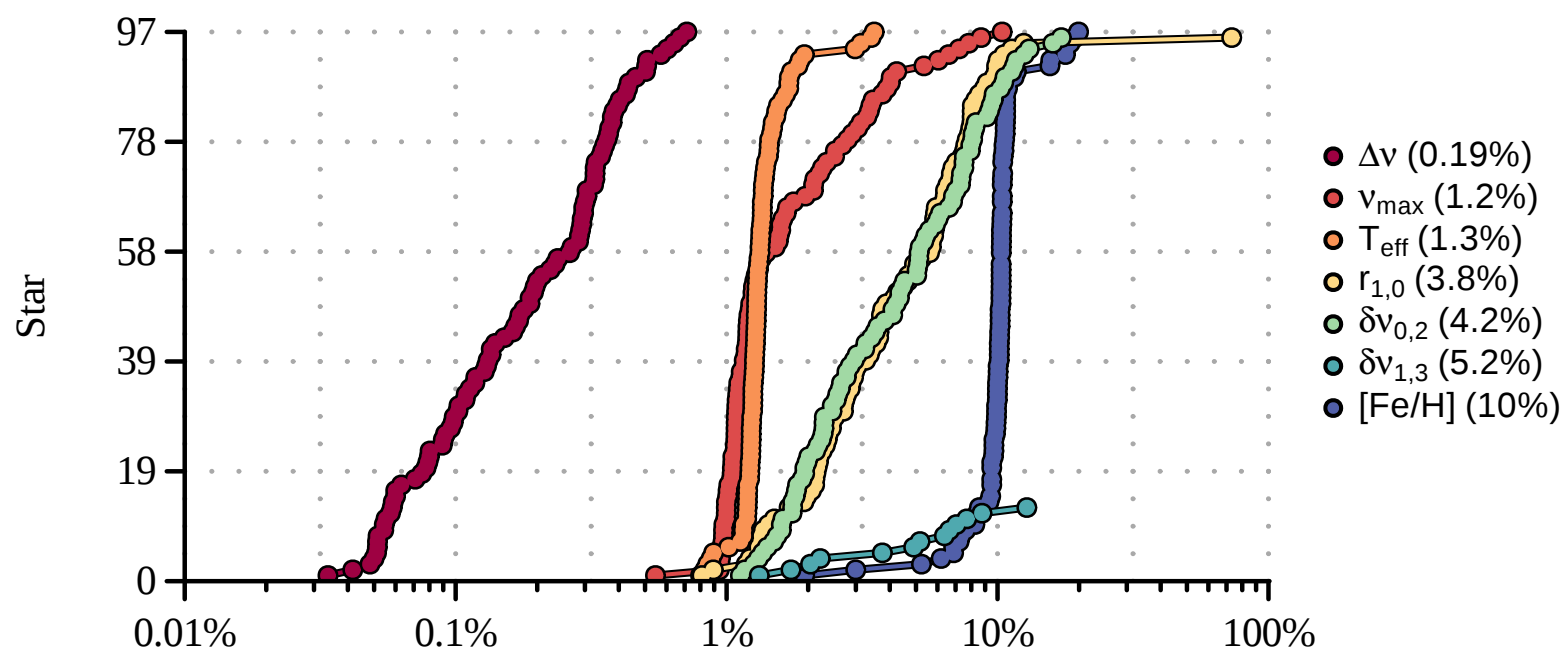

\section{Relative Uncertainty}

Fig. 5. Relative uncertainty of each measurement for each of the 97 stars analyzed here. The uncertainties are given in the sense of $\sigma / \mu$ where $\mu$ is the mean of the estimate and $\sigma$ is the standard deviation - except in the case of $[\mathrm{Fe} / \mathrm{H}]$, where these quantities are instead given for $\exp ([\mathrm{Fe} / \mathrm{H}])$. For each type of measurement, the order of the stars is given in order of increasing relative uncertainty, so for example Star 1 is not necessarily the same star in all the different types of measurements. We note that $\delta v_{1,3}$ measurements are only available for 13 of the stars.

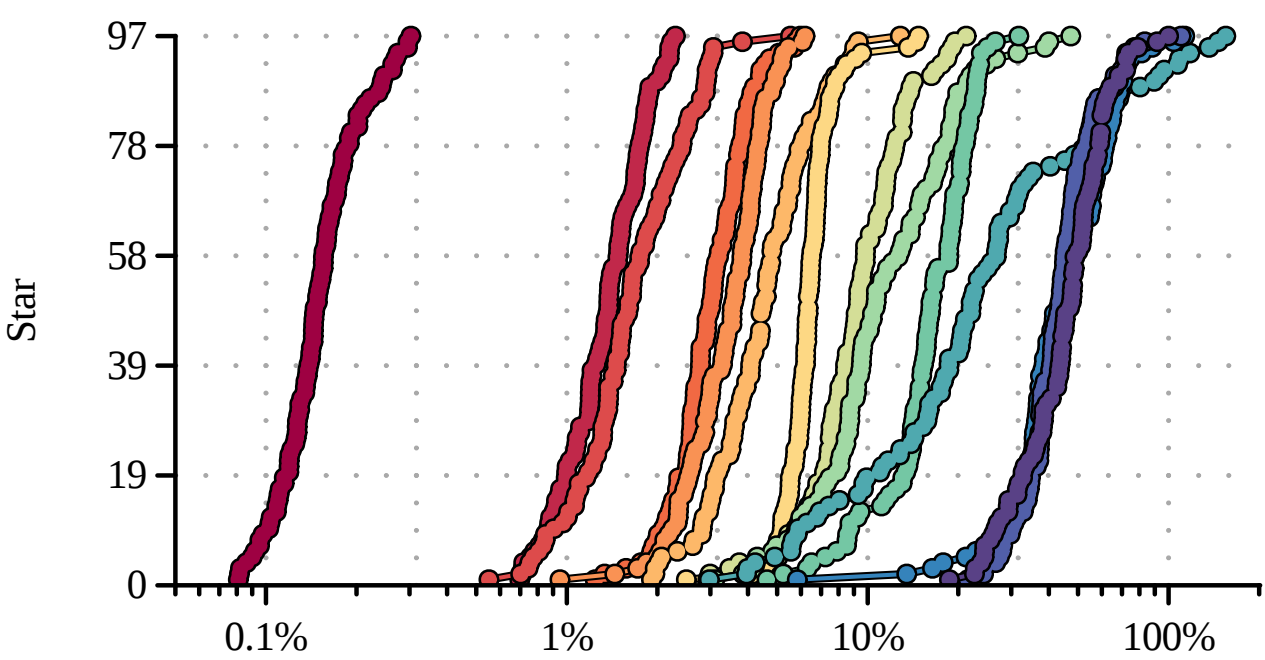

\section{Relative Uncertainty}

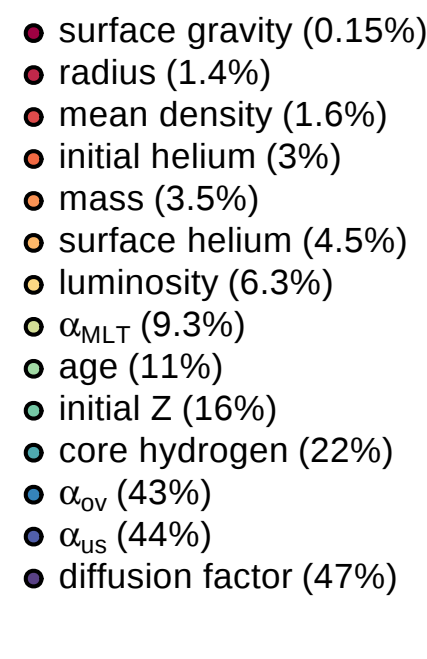

Fig. 6. Cumulative distributions showing the relative uncertainty of each estimated stellar parameter for each of the 97 stars, obtained by running the data in Fig. 5 through SPI.

\subsection{Comparison with Gaia data}

The accuracy of SPI can be gauged by comparing its predictions with observations. In Fig. 8 we show a comparison of predicted luminosities and radii to those from Gaia DR2. Again we find good agreement, despite the systematic errors that may currently be present in the Gaia data. Sahlholdt \& Silva Aguirre (2018) recently compared their predicted radii to Gaia radii for these stars as well and found similar results, although our estimates are in somewhat better agreement, in the sense that we find fewer discrepant stars. Most likely this is due to the fact that our models incorporate a greater variety of mixing length parameters, which is important for determining stellar radii. We furthermore compare the results from SPI to the BASTA models for these stars (Silva Aguirre et al. 2015, 2017) in Fig. 9, finding good agreement.

\subsection{Testing the seismic scaling relations}

The stellar parameters we derive using SPI can be used to check the validity of the seismic scaling relations (Eqs. (1)-(3)). Figure 10 shows a comparison between the masses, radii, and mean densities obtained in these two ways. Overall, there is good agreement, albeit with some significant outliers. We note that two of the discrepant stars - KIC 8760414 and KIC 7106245 (labels 8 and 10 in the figure) - are two of the lowest mass stars and are by far the most metal poor stars of the sample, with $[\mathrm{Fe} / \mathrm{H}]$ values of approximately $-0.9 \mathrm{dex}$ and $-1 \mathrm{dex}$, respectively. In contrast, the next most metal poor star has a metallicity of approximately -0.5 dex (cf. Fig. 3). However, we find no apparent metallicity dependence in the residuals of any of the scaling relations. We note that the large number of outliers in mean density estimates in Fig. 10 arise mainly from 
Spectral Type
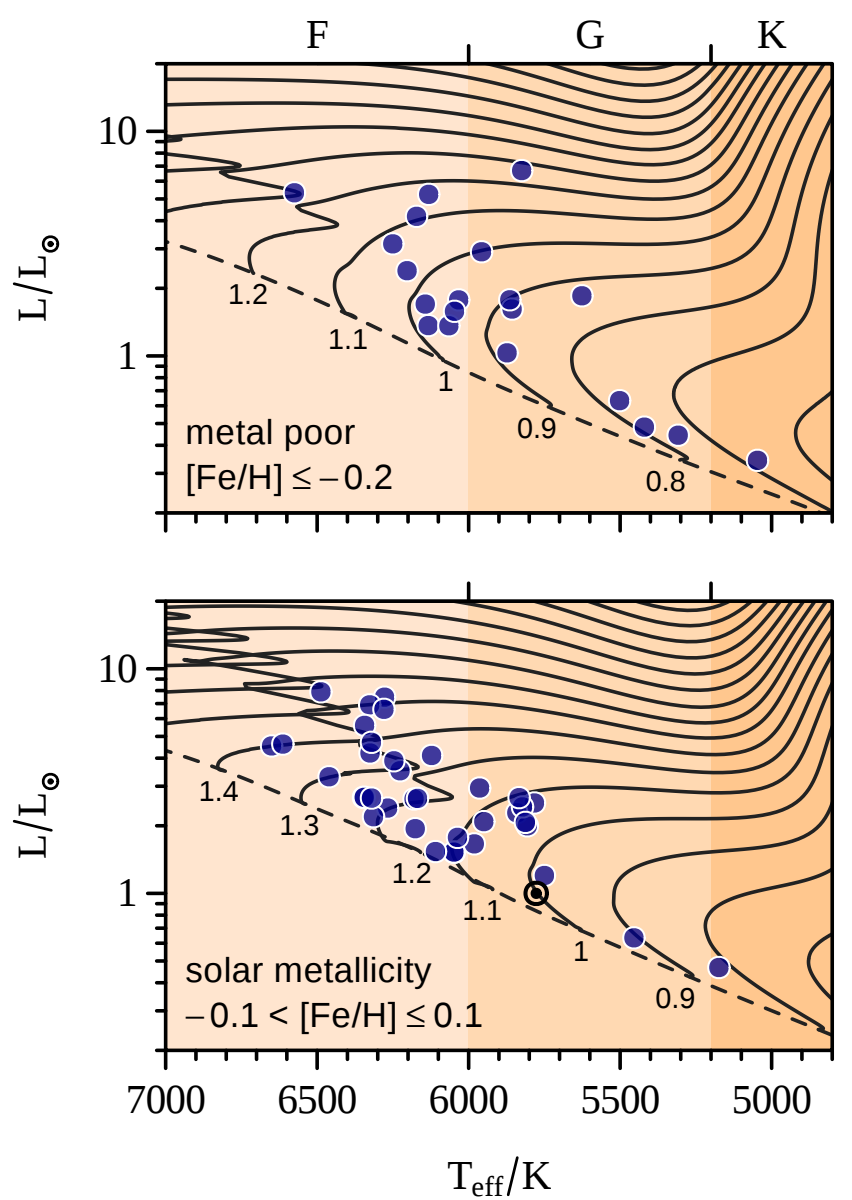

Spectral Type
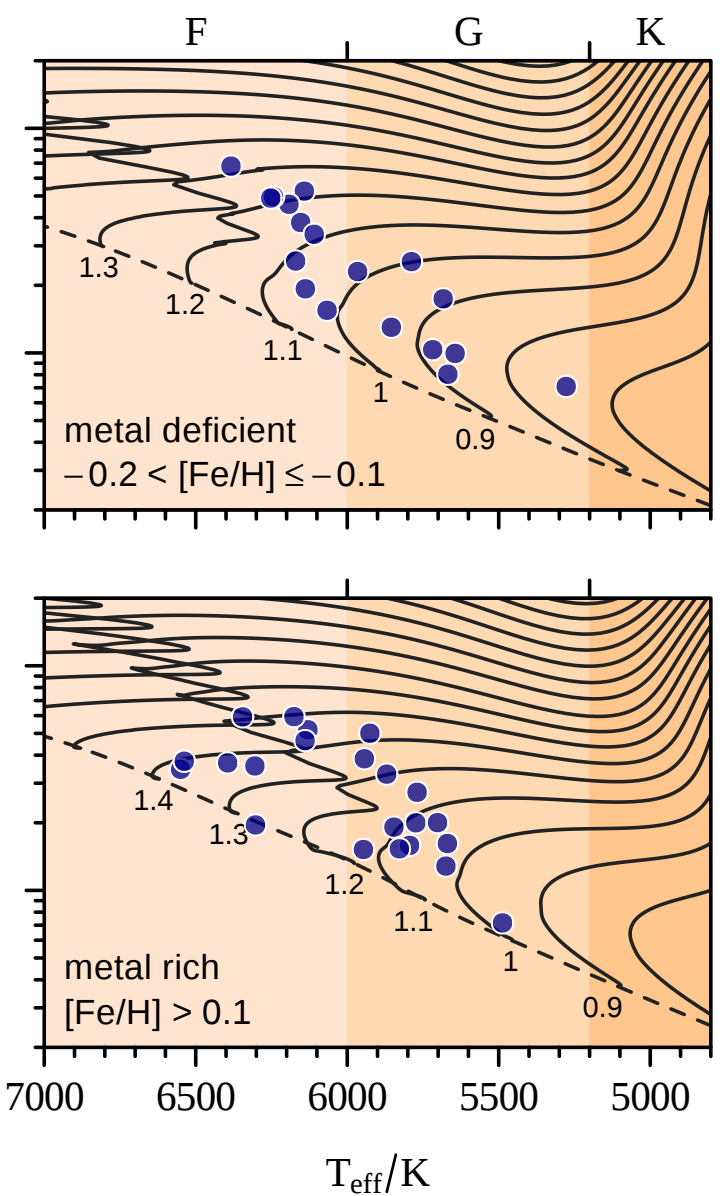

Fig. 7. Hertzsprung-Russell diagrams of solar-like stars with temperatures from spectroscopy and predicted luminosities from asteroseismic modeling. The panels are arranged by metallicity, with the lines showing theoretical evolutionary sequences for stars of the corresponding metallicities having the indicated masses (expressed in solar units). The dashed line traces the zero-age main sequence. The background colors indicate the spectral types of the stars (F, G, K). Typical uncertainties are $0.15 L_{\odot}$ and $100 \mathrm{~K}$.

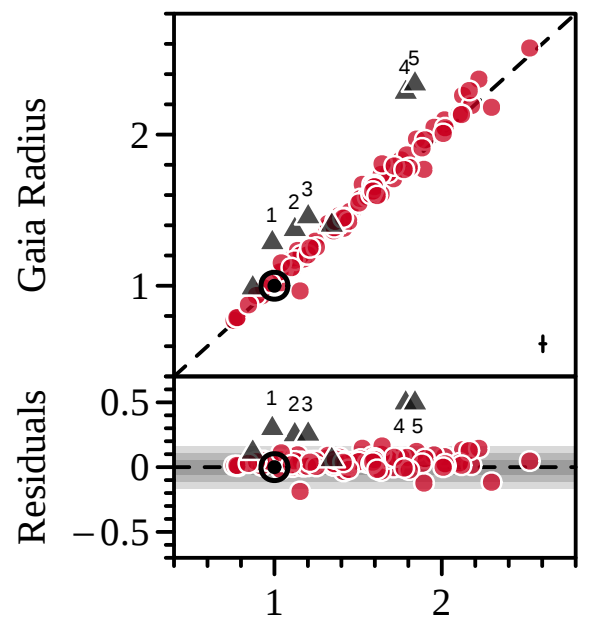

SPI Radius

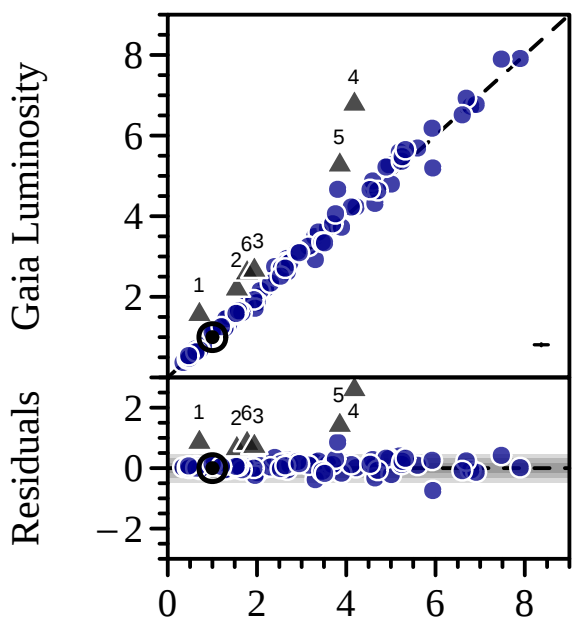

SPI Luminosity

Fig. 8. Comparison of stellar radii (left panel) and luminosities (right panel) in solar units from Gaia observations against those from stellar modeling using the SPI pipeline. Typical uncertainties are shown as error bars in the lower right corners. Each shade of gray in the residual plots represents an average $1 \sigma$, ranging from one to three. Stars with estimates significantly different at the $\sigma>3$ level are shown as gray triangles. Stars which are outliers in more than one panel are labeled. We note the differences in scale between the left and right panels. The biases in the residuals are consistent with zero $\left(-0.056 \pm 0.091 R_{\odot}\right.$ and $\left.-0.14 \pm 0.36 L_{\odot}\right)$ 


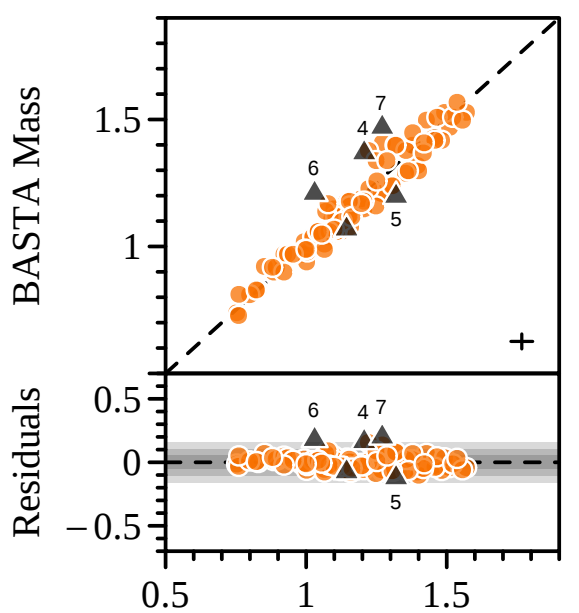

SPI Mass

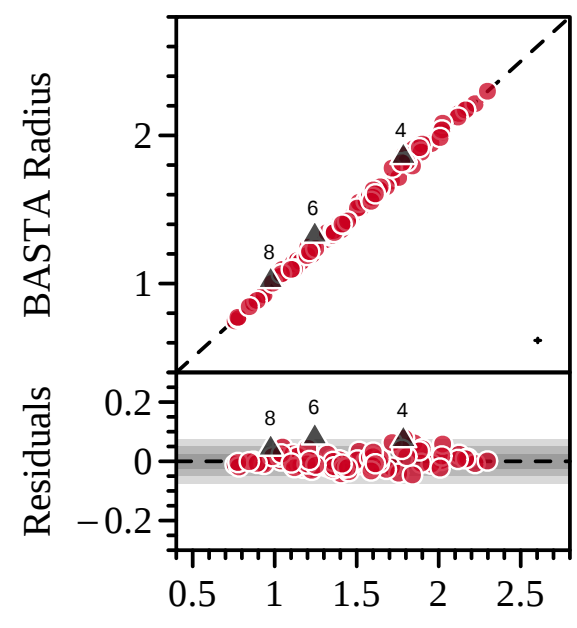

SPI Radius

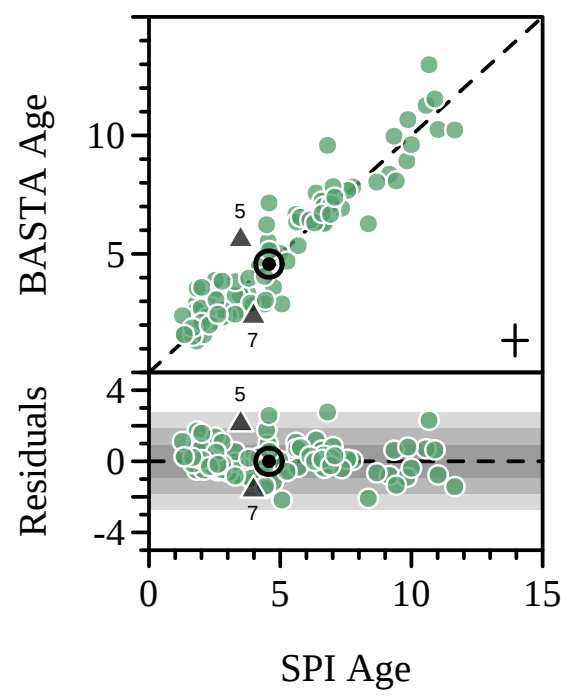

Fig. 9. Comparison of stellar masses (in solar units), radii (in solar units), and ages (in Gyr) determined via stellar modeling using BASTA against those from stellar modeling using SPI. The biases in the residuals are all consistent with zero $\left(0.001 \pm 0.061 M_{\odot},-0.001 \pm 0.027 R_{\odot}\right.$, and $-0.1 \pm 1.0 \mathrm{Gyr})$.
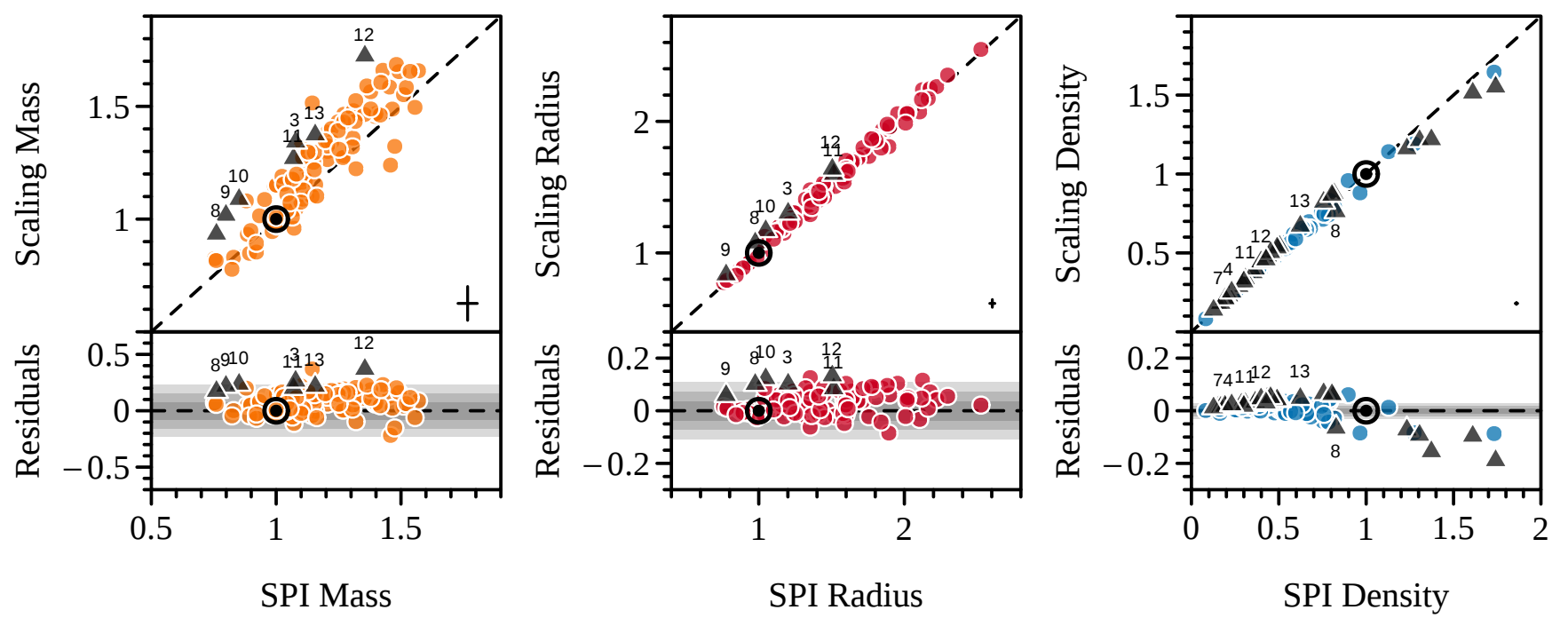

Fig. 10. Comparison of stellar masses, radii, and mean densities (in solar units) determined via the seismic scaling relations (Eqs. (1)-(3)) against those from stellar modeling using the SPI pipeline. The biases in the residuals are all consistent with zero $\left(0.09 \pm 0.11 M_{\odot}, 0.035 \pm 0.045 R_{\odot}\right.$, and $\left.0.005 \pm 0.042 \rho_{\odot}\right)$.

their very small uncertainties. Only one of the labeled outliers, KIC 8478994 is a confirmed planet host (label 9 in the figure see Table A.1).

\subsection{Testing systematic errors in spectroscopy}

We performed two sets of tests aimed at gauging the robustness of stellar parameters to unreliable spectroscopic parameters. In the first test, we biased all of the spectroscopic measurements by systematically changing their reported metallicities and effective temperatures (both independently and simultaneously). We then measured the extent to which their estimated radii, masses, mean densities, and ages changed with respect to the unperturbed estimates of the stellar parameter. In the second test, we increased the reported random uncertainties for each star and measured the change to the uncertainties of the resulting stellar parameters.

The results of these experiments are shown in Figs. 11, $12,13,14,15$, and 16 . There it can be seen that a systematic error of $0.1 \mathrm{dex}$ in $[\mathrm{Fe} / \mathrm{H}]$ measurements translates on average to differences of only $4 \%, 2 \%$, and $1 \%$ in the resulting stellar ages, masses, and radii, respectively. These differences are smaller than the reported relative uncertainties for these quantities $(\sim 11 \%, 3.5 \%, 1.4 \%)$. Similarly, a systematic error of $100 \mathrm{~K}$ in effective temperature results in relative differences of $6 \%, 2 \%$, and $0.4 \%$. Furthermore, increasing the reported uncertainty of $[\mathrm{Fe} / \mathrm{H}]$ measurements by $0.1 \mathrm{dex}(100 \%)$ increases the uncertainties of stellar ages, masses, and radii on average by only $0.01 \mathrm{Gyr}, 0.02 M_{\odot}$, and $0.01 R_{\odot}$, which are well below the reported uncertainties for these estimates $\left(\sim 0.5 \mathrm{Gyr}, 0.04 M_{\odot}\right.$, $\left.0.02 R_{\odot}\right)$. Similarly, increasing the reported uncertainty of $T_{\text {eff }}$ by $100 \mathrm{~K}$ increases the uncertainties of the estimated stellar parameters by $0.2 \mathrm{Gyr}, 0.01 M_{\odot}$, and $0.003 R_{\odot}$. Thus, even relatively large systematic errors and increases to uncertainty result in essentially the same stellar parameters.

When biasing $[\mathrm{Fe} / \mathrm{H}]$ estimates by more than $0.1 \mathrm{dex}(1 \sigma)$, some of the stellar metallicities went beyond the grid of 

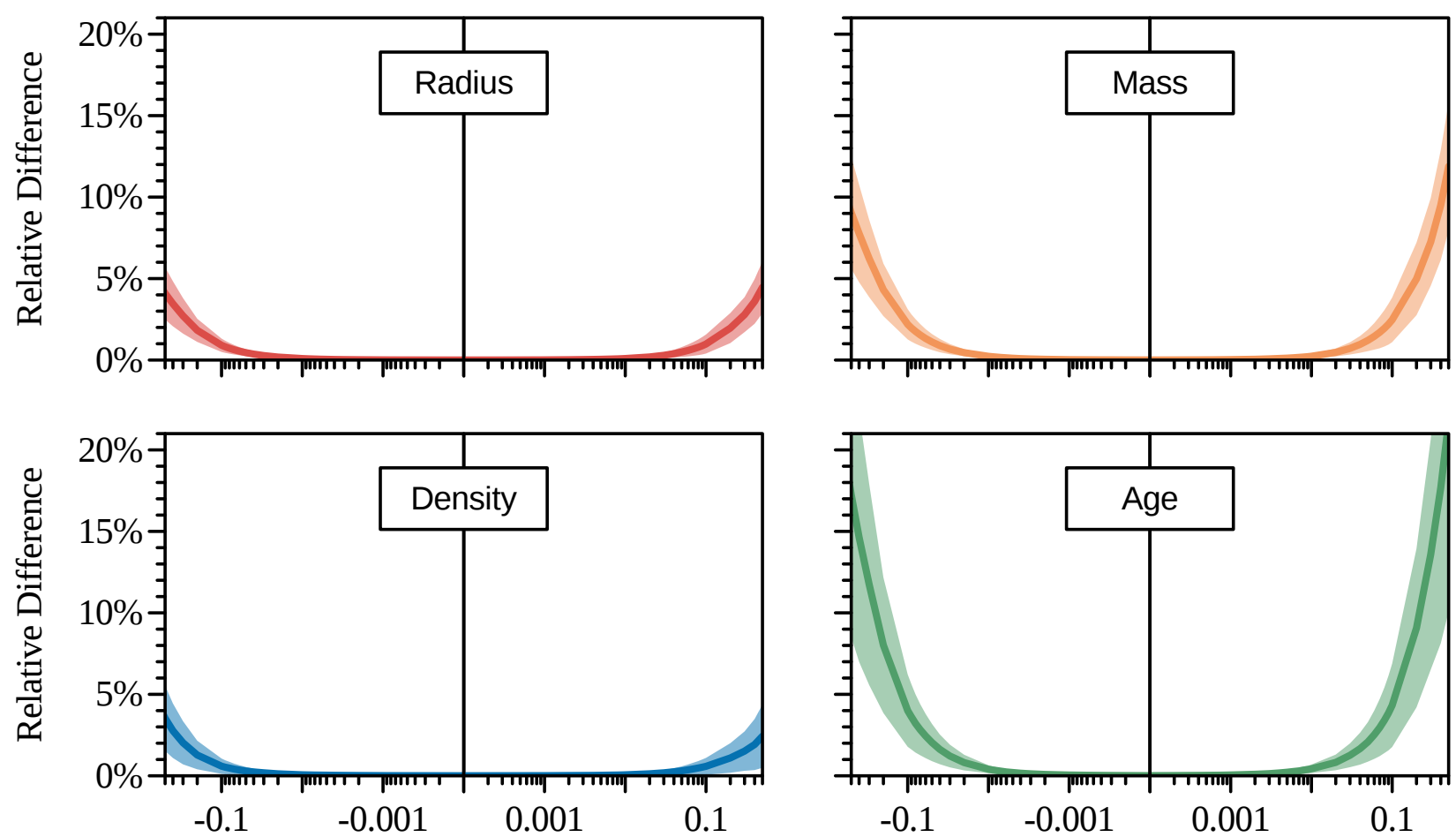

Systematic Error [dex]

$\begin{array}{lll}-0.1 & -0.001 \quad 0.001\end{array}$

0.1

Systematic Error [dex]

Fig. 11. Average relative differences in stellar parameter estimates (radius, mass, density, and stellar age) between estimates made using the reported measurements and estimates made using $[\mathrm{Fe} / \mathrm{H}]$ values that have been biased with systematic errors ranging from $-0.5 \mathrm{dex}$ to $0.5 \mathrm{dex}$. The lines show the mean values and the shaded regions show the standard deviations across the 97 stars.
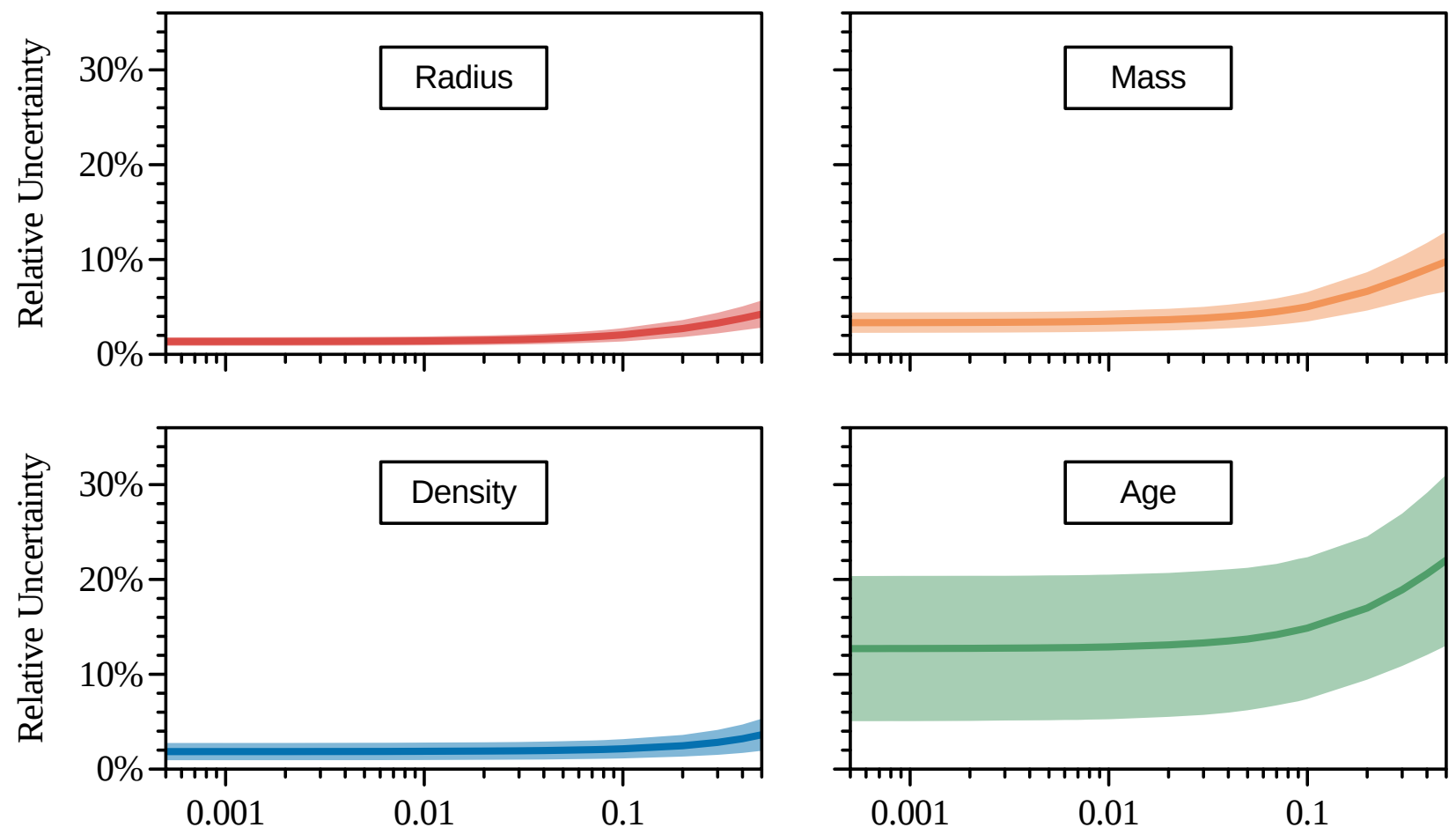

Added Uncertainty [dex]

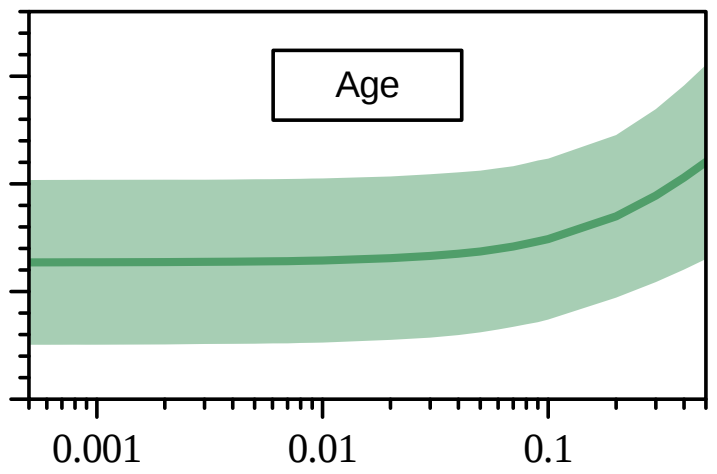

Added Uncertainty [dex]

Fig. 12. Average relative uncertainties in stellar parameter estimates (radius, mass, mean density, and stellar age) as a function of the amount of additional random uncertainty given to $[\mathrm{Fe} / \mathrm{H}]$ measurements ranging logarithmically from 0.0005 dex to 0.5 dex. 
E. P. Bellinger' et al.: The impact of systematic errors in spectroscopic measurements on stellar parameters
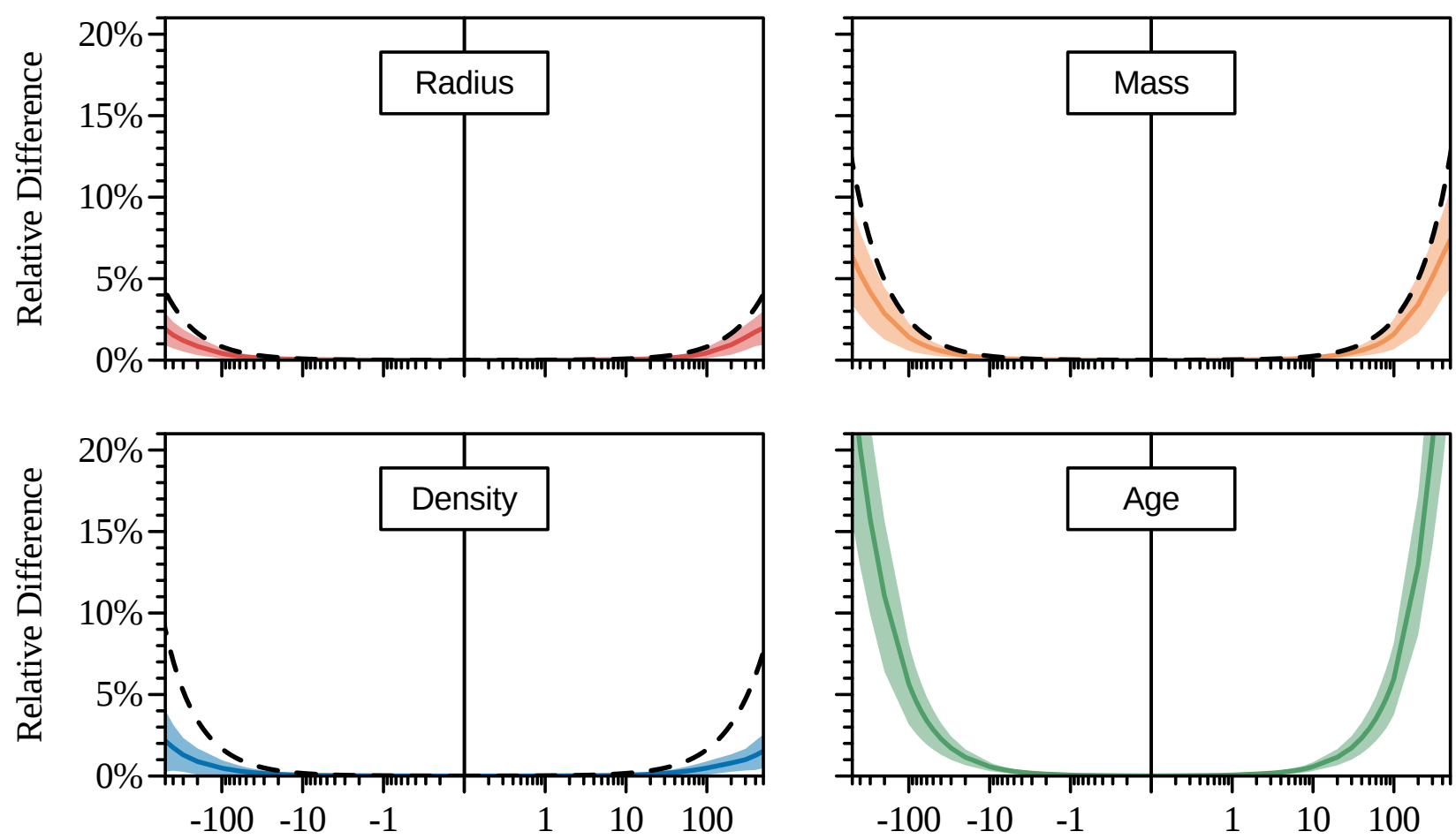

Systematic Error [K]

Systematic Error [K]

Fig. 13. Average relative differences in stellar parameter estimates (radius, mass, mean density, and stellar age) between estimates made using the reported measurements and estimates made using $T_{\text {eff }}$ values that have been biased with systematic errors ranging from $-500 \mathrm{~K}$ to $500 \mathrm{~K}$. The dashed lines show the relative differences from the seismic scaling relations (Eqs. (1)-(3)).
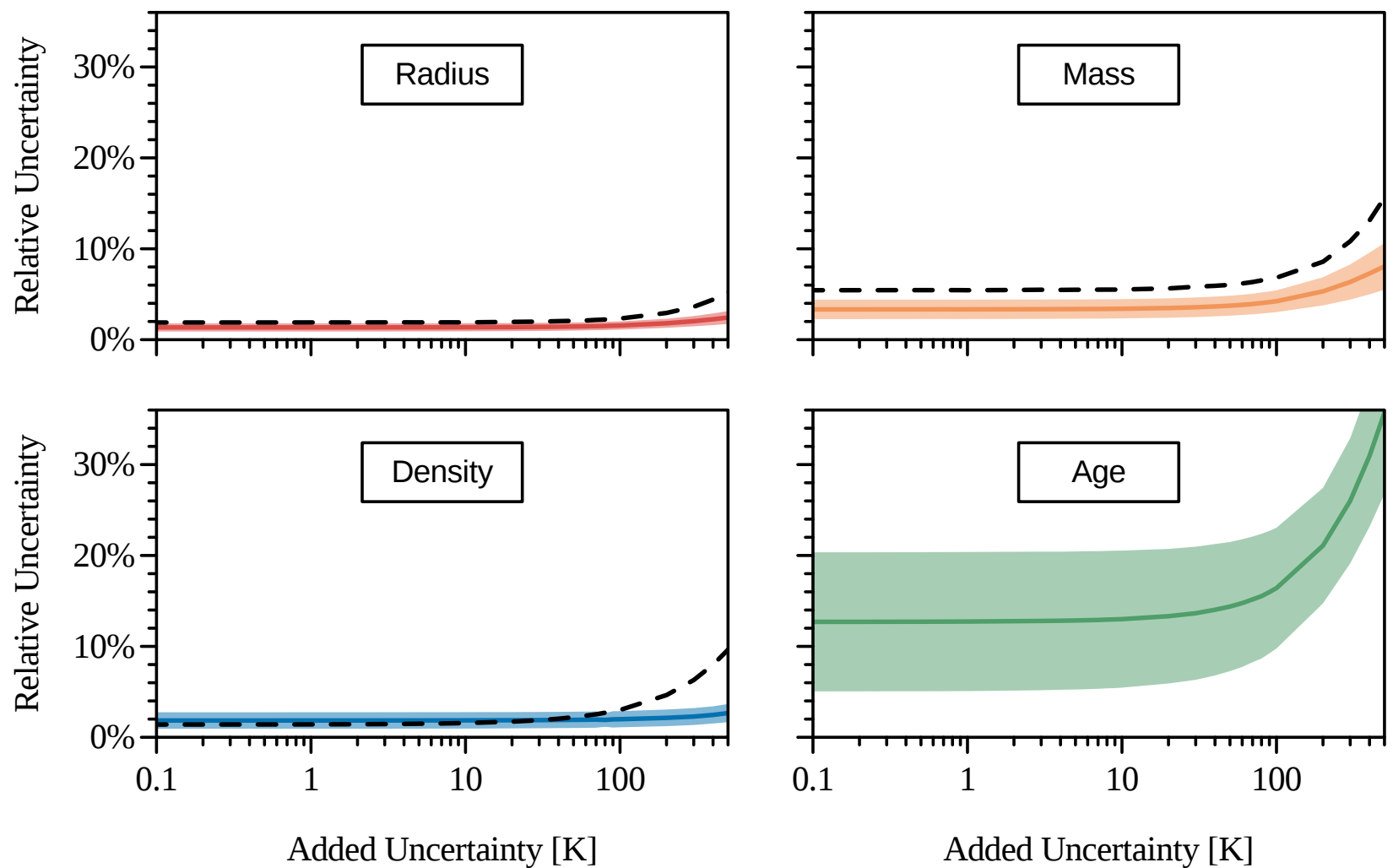

Fig. 14. Average relative uncertainties in stellar parameter estimates (radius, mass, mean density, and stellar age) as a function of the amount of additional random uncertainty given to $T_{\text {eff }}$ measurements ranging logarithmically from $0.1 \mathrm{~K}$ to $500 \mathrm{~K}$. 

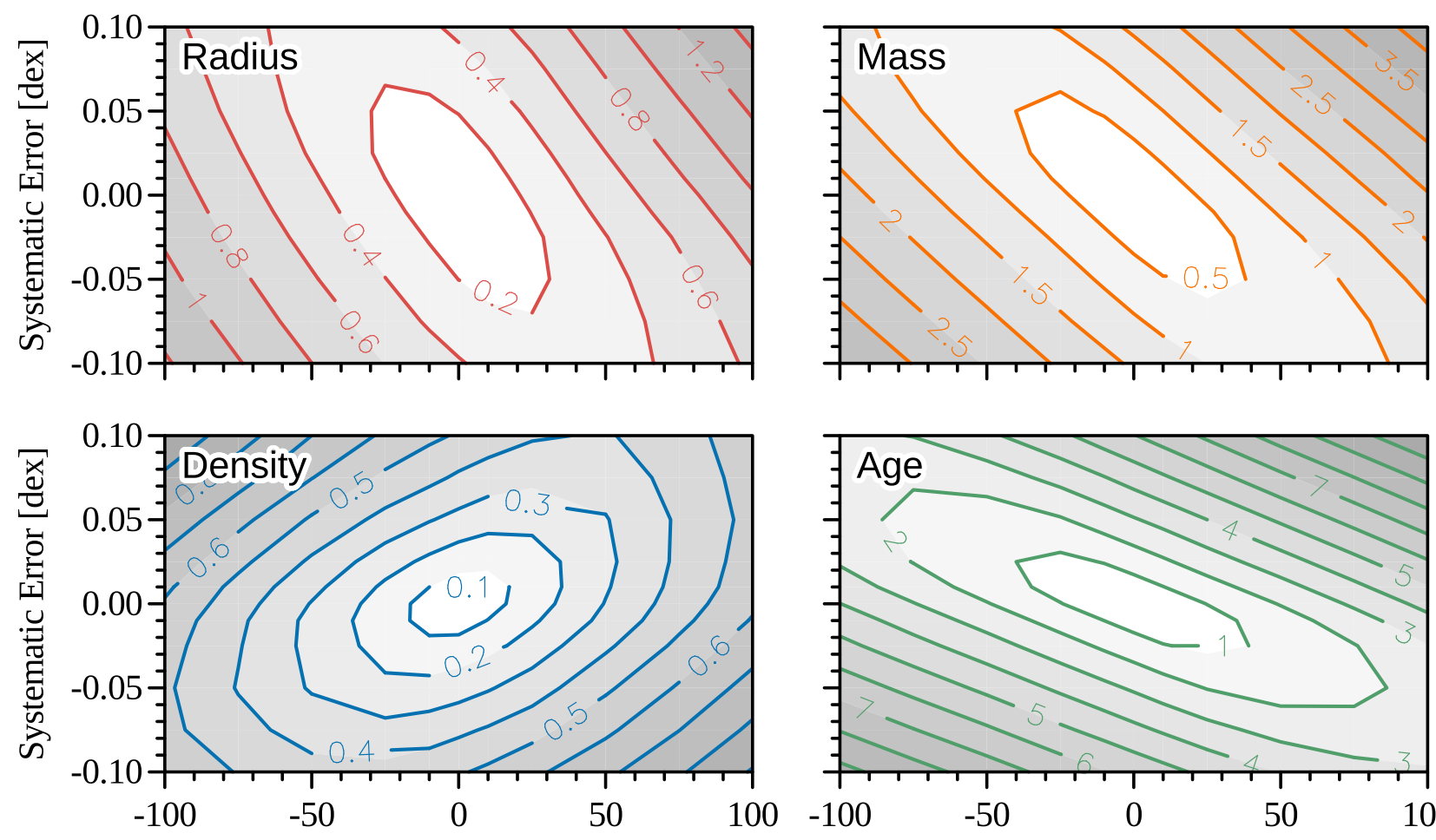

Systematic Error [K]

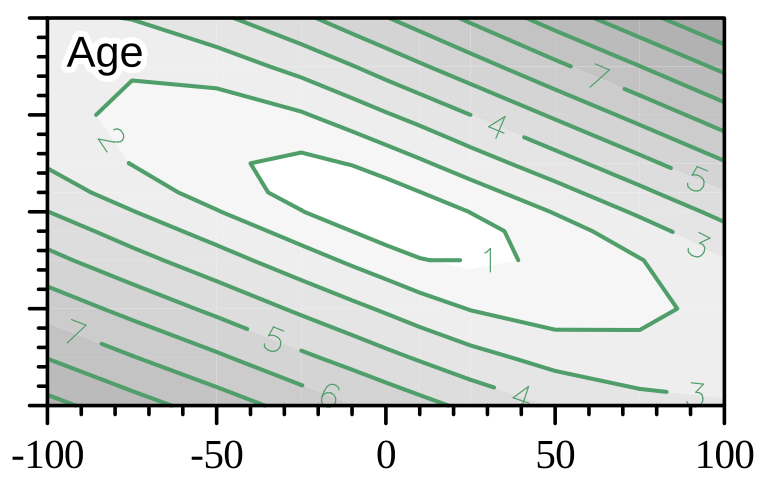

Systematic Error [K]

Fig. 15. Average relative differences (in percent) in stellar parameter estimates (radius, mass, mean density, and stellar age) between estimates made using the reported measurements and estimates made using (simultaneously) systematically biased [Fe/H] and $T_{\text {eff }}$ values.
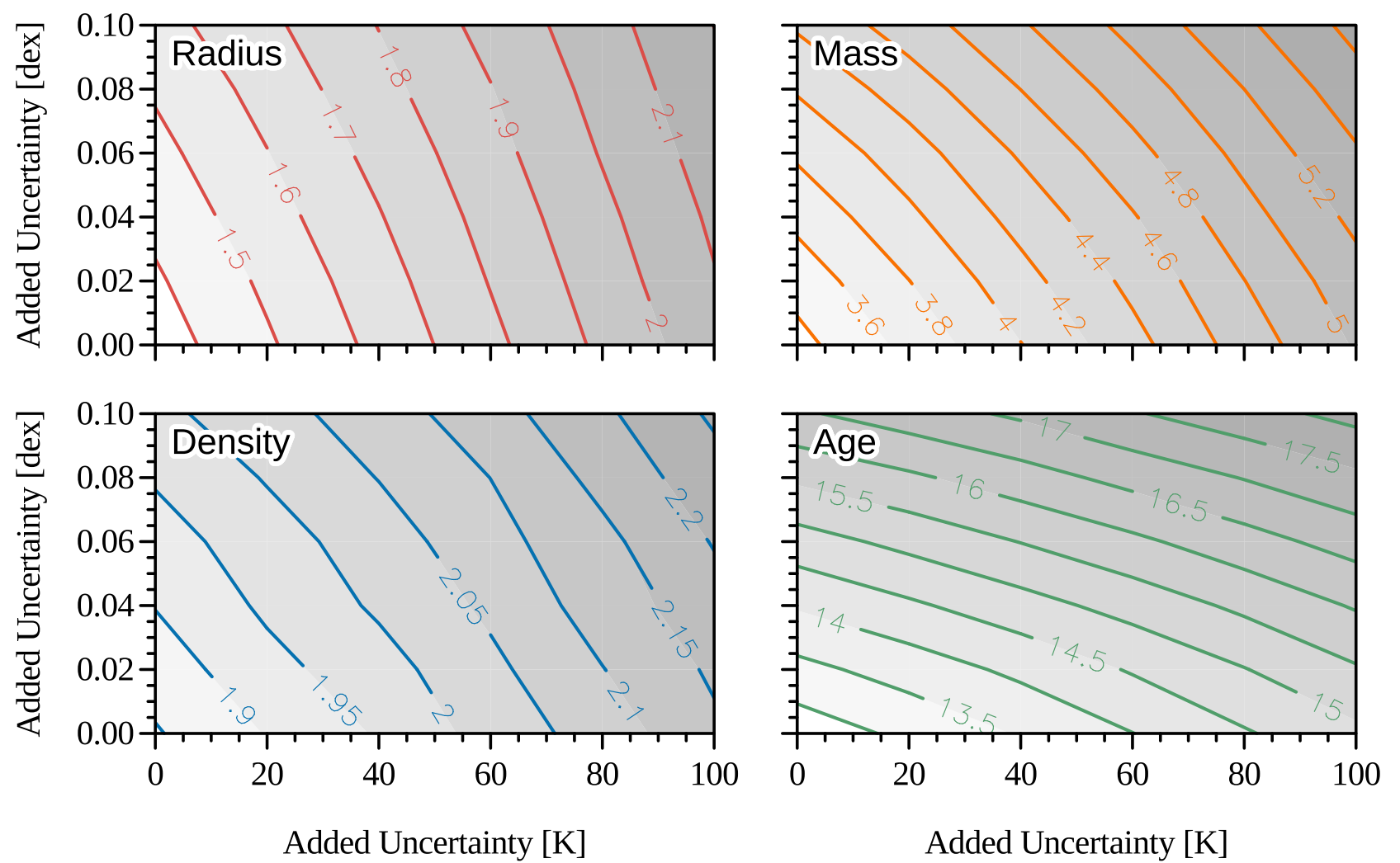

Fig. 16. Average relative uncertainties (in percent) in stellar parameter estimates (radius, mass, mean density, and stellar age) as a function of the amount of additional random uncertainty given to $[\mathrm{Fe} / \mathrm{H}]$ and $T_{\text {eff }}$ measurements. 
models and were dropped. By $0.5 \operatorname{dex}(5 \sigma)$, only half of the stars remained, and at this point we stopped the experiment. We find that in order for the relative differences in the resulting stellar ages, masses, and radii to be equal to their reported uncertainties, $[\mathrm{Fe} / \mathrm{H}]\left(T_{\text {eff }}\right)$ measurements would need to be biased by at least $0.24 \operatorname{dex}(175 \mathrm{~K}), 0.14 \operatorname{dex}(200 \mathrm{~K})$, and $0.14 \operatorname{dex}(300 \mathrm{~K})$, respectively.

The results regarding the uncertainties are consistent with the theoretical findings of Angelou et al. (2017, their Fig. 7). Though our analysis was done using frequency ratios to account for the surface term, Basu \& Kinnane (2018) have similarly shown that how the surface term is accounted for does not affect estimates of the global properties (but see also Aarslev et al. 2017).

The tests regarding effective temperatures also show these biases propagated through the seismic scaling relations (Eqs. (1), (2), (3)). We find that estimates obtained using SPI are even more robust to systematic errors than the scaling relations.

\section{Conclusions}

We estimated the stellar parameters of 97 main-sequence and early sub-giant stars observed by the Kepler spacecraft using the SPI pipeline. We compared the estimates of stellar mass, radius, and mean density that we obtained from stellar modeling for these stars to the estimates from seismic scaling relations and found good agreement. We found no evidence in the residuals for a trend with metallicity. We similarly compared the estimates of stellar radius and luminosity that we obtained from stellar modeling to the measurements from Gaia DR2, and again found good agreement.

We used these stars to test the impact of systematic errors in metallicity and effective temperature on the determination of stellar age, mass, and radius via asteroseismic stellar modeling. We found that the resulting stellar parameters from SPI are stable with respect to even relatively large systematic errors in the spectroscopic measurements. We emphasize that these results are only valid for asteroseismic modeling performed using SPI; results from other methods may vary. We note that we have not tested the impact on more evolved stars, such as red giants, and there too the results may differ. We note also that there may be other sources of systematic errors in the theoretical models, such as the assumed opacities, nuclear reaction rates, and mixture of metals, which we have not examined here.

These results should not be taken to imply that spectroscopic measurements are unimportant. As was shown by Bellinger et al. (2016) and Angelou et al. (2017), metallicity measurements are consistently rated as being highly important for constraining stellar models, as they provide unique information that is not redundant with the oscillation data. However, measurements of stellar metallicity are most important in constraining aspects of the models related to the initial chemical composition and mixing processes that take place during the evolution. They are, however, comparatively less important in constraining global parameters such as stellar age, mass, and radius from existing stellar models, as these quantities are well-constrained by seismology.

We conclude that asteroseismic modeling is a very reliable technique for obtaining ages, masses, radii, and other parameters of solar-like oscillators on the main sequence. Consequently, the parameters for their exoplanets, being that they are based on these values, are also robust to such systematics.

Acknowledgements. We thank Didier Queloz and Carla Wiles for useful discussions. We thank the anonymous referee for their feedback, which improved the manuscript. We also thank the developers of the astroquery package
(Ginsburg et al. 2018) and the NASA Exoplanet Archive (Akeson et al. 2013). Funding for the Stellar Astrophysics Centre is provided by The Danish National Research Foundation (Grant agreement no.: DNRF106). The research leading to the presented results has received funding from the European Research Council under the European Community's Seventh Framework Programme (FP7/20072013)/ERC grant agreement no 338251 (StellarAges). S.B. acknowledges partial support from NSF grant AST-1514676 and NASA grant NNX13AE70G.

\section{References}

Aarslev, M. J., Christensen-Dalsgaard, J., Lund, M. N., Silva Aguirre, V., \& Gough, D. 2017, Eur. Phys. J. Web Conf., 160, 03010

Adibekyan, V., Sousa, S. G., \& Santos, N. C. 2018, Asteroseismol. Exoplanets: Listen. Stars Search. New Worlds, 49, 225

Akeson, R. L., Chen, X., Ciardi, D., et al. 2013, PASP, 125, 989

Anders, F., Chiappini, C., Rodrigues, T. S., et al. 2017, A\&A, 597, A30

Andrae, R., Fouesneau, M., Creevey, O., et al. 2018, A\&A, 616, A8

Angelou, G. C., Bellinger, E. P., Hekker, S., \& Basu, S. 2017, ApJ, 839, 116

Barclay, T., Rowe, J. F., Lissauer, J. J., et al. 2013, Nature, 494, 452

Basu, S. 1997, MNRAS, 288, 572

Basu, S., \& Chaplin, W. 2017, Asteroseismic Data Analysis: Foundations and Techniques, Princeton Series in Modern Obs (Princeton, NJ: Princeton University Press)

Basu, S., \& Kinnane, A. 2018, ApJ, 869, 8

Batalha, N. M., Borucki, W. J., Bryson, S. T., et al. 2011, ApJ, 729, 27

Belkacem, K., Goupil, M. J., Dupret, M. A., et al. 2011, A\&A, 530, A142

Bellinger, E. P., Angelou, G. C., Hekker, S., et al. 2016, ApJ, 830, 31

Bellinger, E. P., Basu, S., Hekker, S., \& Ball, W. H. 2017, ApJ, 851, 80

Borucki, W. J., Koch, D., Basri, G., et al. 2010a, Science, 327, 977

Borucki, W. J., Koch, D. G., Brown, T. M., et al. 2010b, ApJ, 713, L126

Breiman, L. 2001, Mach. Learn., 45, 5

Brown, T. M., Gilliland, R. L., Noyes, R. W., \& Ramsey, L. W. 1991, ApJ, 368, 599

Brown, T. M., Christensen-Dalsgaard, J., Weibel-Mihalas, B., \& Gilliland, R. L. 1994, ApJ, 427, 1013

Campante, T. L., Barclay, T., Swift, J. J., et al. 2015, ApJ, 799, 170

Campante, T. L., Lund, M. N., Kuszlewicz, J. S., et al. 2016, ApJ, 819, 85

Carter, J. A., Agol, E., Chaplin, W. J., et al. 2012, Science, 337, 556

Chaplin, W. J., \& Miglio, A. 2013, ARA\&A, 51, 353

Chaplin, W. J., Sanchis-Ojeda, R., Campante, T. L., et al. 2013, ApJ, 766, 101

Chiappini, C., Anders, F., Rodrigues, T. S., et al. 2015, A\&A, 576, L12

Christensen-Dalsgaard, J. 1984, in Space Research in Stellar Activity and Variability, eds. A. Mangeney, \& F. Praderie, 11

Christensen-Dalsgaard, J., \& Silva Aguirre, V. 2018, in Handbook of Exoplanets, eds. H. J. Deeg, \& J. A. Belmonte (Berlin, Heidelberg: Springer), 18

Cochran, W. D., Hatzes, A. P., Butler, R. P., \& Marcy, G. W. 1997, ApJ, 483, 457 Creevey, O. L., Thévenin, F., Basu, S., et al. 2013, MNRAS, 431, 2419

Davies, G. R., Silva Aguirre, V., Bedding, T. R., et al. 2016, MNRAS, 456, 2183

Deheuvels, S., Brandão, I., Silva Aguirre, V., et al. 2016, A\&A, 589, A93

Feuillet, D. K., Bovy, J., Holtzman, J., et al. 2018, MNRAS, 477, 2326

Fressin, F., Torres, G., Désert, J.-M., et al. 2011, ApJS, 197, 5

Friedman, J., Hastie, T., \& Tibshirani, R. 2001, The Elements of Statistical Learning (New York: Springer), 1

Gai, N., Basu, S., Chaplin, W. J., \& Elsworth, Y. 2011, ApJ, 730, 63

Gaia Collaboration (Brown, A. G. A., et al.) 2018, A\&A, 616, A1

Gaulme, P., McKeever, J., Jackiewicz, J., et al. 2016, ApJ, 832, 121

Geurts, P., Ernst, D., \& Wehenkel, L. 2006, Mach. Learn., 63, 3

Gilliland, R. L., Marcy, G. W., Rowe, J. F., et al. 2013, ApJ, 766, 40

Ginsburg, A., Sipocz, B., Parikh, M., et al. 2018, astropy/astroquery: v0.3.7 release

Gratia, P., \& Fabrycky, D. 2017, MNRAS, 464, 1709

Guggenberger, E., Hekker, S., Basu, S., \& Bellinger, E. 2016, MNRAS, 460, 4277

Guggenberger, E., Hekker, S., Angelou, G. C., Basu, S., \& Bellinger, E. P. 2017, MNRAS, 470, 2069

Guillochon, J., Ramirez-Ruiz, E., \& Lin, D. 2011, ApJ, 732, 74

Hadden, S., \& Lithwick, Y. 2014, ApJ, 787, 80

Han, E., Wang, S. X., Wright, J. T., et al. 2014, PASP, 126, 827

Haywood, R. D., Vanderburg, A., Mortier, A., et al. 2018, AJ, 155, 203

Hekker, S., Elsworth, Y., Mosser, B., et al. 2013, A\&A, 556, A59

Hjørringgaard, J. G., Silva Aguirre, V., White, T. R., et al. 2017, MNRAS, 464, 3713

Howell, S. B., Rowe, J. F., Bryson, S. T., et al. 2012, ApJ, 746, 123

Huber, D. 2016, IAU Focus Meet., 29, 620

Huber, D. 2018, Asteroseismol. Exoplanets: Listen. Stars Search. New Worlds, 49, 119

Huber, D., Bedding, T. R., Stello, D., et al. 2011, ApJ, 743, 143 
Huber, D., Carter, J. A., Barbieri, M., et al. 2013, Science, 342, 331

Huber, D., Silva Aguirre, V., Matthews, J. M., et al. 2014, ApJS, 211, 2

Huber, D., Zinn, J., Bojsen-Hansen, M., et al. 2017, ApJ, 844, 102

Jenkins, J. M., Twicken, J. D., Batalha, N. M., et al. 2015, AJ, 150, 56

Kamiaka, S., Benomar, O., \& Suto, Y. 2018, MNRAS, 479, 391

Kjeldsen, H., \& Bedding, T. R. 1995, A\&A, 293, 87

Königl, A., Giacalone, S., \& Matsakos, T. 2017, ApJ, 846, L13

Lai, D., Foucart, F., \& Lin, D. N. C. 2011, MNRAS, 412, 2790

Lebreton, Y., \& Goupil, M. J. 2014, A\&A, 569, A21

Li, G., \& Winn, J. N. 2016, ApJ, 818, 5

Lin, D. N. C., Bodenheimer, P., \& Richardson, D. C. 1996, Nature, 380, 606

Lund, M. N., Silva Aguirre, V., Davies, G. R., et al. 2017, ApJ, 835, 172

Lundkvist, M. S., Huber, D., Silva Aguirre, V., \& Chaplin, W. J. 2018, in Handbook of Exoplanets, eds. H. J. Deeg, \& J. A. Belmonte (Berlin, Heidelberg: Springer), 24

Marcy, G. W., Isaacson, H., Howard, A. W., et al. 2014, ApJS, 210, 20

Marrese, P. M., Marinoni, S., Fabrizio, M., \& Altavilla, G. 2019, A\&A, 621, A144

Massey, P., \& Hanson, M. M. 2013, Astronomical Spectroscopy (Berlin, Heidelberg: Springer), 35

Mathur, S., Metcalfe, T. S., Woitaszek, M., et al. 2012, ApJ, 749, 152

Matsakos, T., \& Königl, A. 2015, ApJ, 809, L20

Matsakos, T., \& Königl, A. 2017, AJ, 153, 60

Metcalfe, T. S., Creevey, O. L., Doğan, G., et al. 2014, ApJS, 214, 27

Morton, T. D., \& Johnson, J. A. 2011, ApJ, 729, 138

Mosser, B., Michel, E., Belkacem, K., et al. 2013, A\&A, 550, A126

Nissen, P. E., Silva Aguirre, V., Christensen-Dalsgaard, J., et al. 2017, A\&A, 608, A112

Pál, A., Bakos, G. Á., Torres, G., et al. 2008, ApJ, 680, 1450

Paxton, B., Bildsten, L., Dotter, A., et al. 2011, ApJS, 192, 3

Paxton, B., Cantiello, M., Arras, P., et al. 2013, ApJS, 208, 4

Paxton, B., Marchant, P., Schwab, J., et al. 2015, ApJS, 220, 15

Paxton, B., Schwab, J., Bauer, E. B., et al. 2018, ApJS, 234, 34

Plavchan, P., \& Bilinski, C. 2013, ApJ, 769, 86

Prša, A., Harmanec, P., Torres, G., et al. 2016, AJ, 152, 41
Rogers, L. A. 2015, ApJ, 801, 41

Rowe, J. F., Bryson, S. T., Marcy, G. W., et al. 2014, ApJ, 784, 45

Roxburgh, I. W., \& Vorontsov, S. V. 2003, A\&A, 411, 215

Safonova, M., Murthy, J., \& Shchekinov, Y. A. 2016, Int. J. Astrobiol., 15, 93

Sahlholdt, C. L., \& Silva Aguirre, V. 2018, MNRAS, 481, L125

Seager, S., \& Mallén-Ornelas, G. 2003, ApJ, 585, 1038

Seager, S., Kuchner, M., Hier-Majumder, C. A., \& Militzer, B. 2007, ApJ, 669, 1279

Sharma, S., Stello, D., Bland-Hawthorn, J., Huber, D., \& Bedding, T. R. 2016, ApJ, 822, 15

Silva Aguirre, V., Bojsen-Hansen, M., Slumstrup, D., et al. 2018, MNRAS, 475, 5487

Silva Aguirre, V., Davies, G. R., Basu, S., et al. 2015, MNRAS, 452, 2127

Silva Aguirre, V., Lund, M. N., Antia, H. M., et al. 2017, ApJ, 835, 173

Škoda, P. 2017, Proceedings of EURO-VO Workshop Astronomical Spectroscopy and Virtual Observatory

Steffen, J. H., Fabrycky, D. C., Ford, E. B., et al. 2012, MNRAS, 421, 2342

Steffen, J. H., Fabrycky, D. C., Agol, E., et al. 2013, MNRAS, 428, 1077

Teyssandier, J., Terquem, C., \& Papaloizou, J. C. B. 2013, MNRAS, 428, 658

Themeß1, N., Hekker, S., Southworth, J., et al. 2018, MNRAS, 478, 4669

Thies, I., Kroupa, P., Goodwin, S. P., Stamatellos, D., \& Whitworth, A. P. 2011, MNRAS, 417, 1817

Torres, G., Andersen, J., \& Giménez, A. 2010, A\&ARv, 18, 67

Townsend, R. H. D., \& Teitler, S. A. 2013, MNRAS, 435, 3406

Ulrich, R. K. 1986, ApJ, 306, L37

Valle, G., Dell'Omodarme, M., Prada Moroni, P. G., \& Degl'Innocenti, S. 2018, A\&A, 620, A168

Van Eylen, V., \& Albrecht, S. 2015, ApJ, 808, 126

Van Eylen, V., Lund, M. N., Silva Aguirre, V., et al. 2014, ApJ, 782, 14

Viani, L. S., Basu, S., Chaplin, W. J., Davies, G. R., \& Elsworth, Y. 2017, ApJ, 843,11

Watson, C. A., Littlefair, S. P., Diamond, C., et al. 2011, MNRAS, 413, L71

Weiss, L. M., \& Marcy, G. W. 2014, ApJ, 783, L6

White, T. R., Bedding, T. R., Stello, D., et al. 2011, ApJ, 743, 161

Xie, J.-W. 2014, ApJS, 210, 25 
E. P. Bellinger' et al.: The impact of systematic errors in spectroscopic measurements on stellar parameters

\section{Appendix A: Stellar parameters}

Table A.1. Stellar parameters for the Kepler Ages and LEGACY stars from the SPI pipeline.

\begin{tabular}{|c|c|c|c|c|c|c|}
\hline $\mathrm{KIC}$ & $\tau / \mathrm{Gyr}$ & $M / M_{\odot}$ & $R / R_{\odot}$ & $\rho / \rho_{\odot}$ & $L / L_{\odot}$ & Planets \\
\hline 1435467 & $2.03 \pm 0.37$ & $1.396 \pm 0.033$ & $1.712 \pm 0.018$ & $0.2789 \pm 0.0045$ & $4.22 \pm 0.21$ & \\
\hline 2837475 & $1.34 \pm 0.51$ & $1.455 \pm 0.061$ & $1.638 \pm 0.022$ & $0.331 \pm 0.010$ & $4.62 \pm 0.30$ & \\
\hline 3425851 & $3.77 \pm 0.58$ & $1.145 \pm 0.049$ & $1.354 \pm 0.020$ & $0.4625 \pm 0.0062$ & $2.67 \pm 0.17$ & \\
\hline 3427720 & $2.34 \pm 0.23$ & $1.108 \pm 0.032$ & $1.121 \pm 0.010$ & $0.7904 \pm 0.0079$ & $1.527 \pm 0.079$ & \\
\hline 3456181 & $2.62 \pm 0.37$ & $1.428 \pm 0.062$ & $2.125 \pm 0.035$ & $0.1489 \pm 0.0027$ & $6.79 \pm 0.38$ & \\
\hline 3544595 & $6.63 \pm 0.64$ & $0.885 \pm 0.038$ & $0.924 \pm 0.016$ & $1.128 \pm 0.025$ & $0.804 \pm 0.058$ & $2^{a}$ \\
\hline 3632418 & $3.40 \pm 0.37$ & $1.271 \pm 0.047$ & $1.849 \pm 0.026$ & $0.2008 \pm 0.0020$ & $4.59 \pm 0.33$ & $1^{b}$ \\
\hline 3656476 & $9.16 \pm 0.80$ & $1.079 \pm 0.027$ & $1.318 \pm 0.017$ & $0.4749 \pm 0.0095$ & $1.62 \pm 0.11$ & \\
\hline 3735871 & $1.81 \pm 0.26$ & $1.108 \pm 0.032$ & $1.109 \pm 0.010$ & $0.816 \pm 0.014$ & $1.535 \pm 0.063$ & \\
\hline 4141376 & $3.48 \pm 0.56$ & $0.993 \pm 0.041$ & $1.038 \pm 0.013$ & $0.897 \pm 0.031$ & $1.365 \pm 0.094$ & $1^{c}$ \\
\hline 4143755 & $10.56 \pm 0.52$ & $0.898 \pm 0.019$ & $1.414 \pm 0.013$ & $0.3211 \pm 0.0036$ & $1.85 \pm 0.11$ & \\
\hline 4349452 & $2.47 \pm 0.55$ & $1.206 \pm 0.055$ & $1.312 \pm 0.023$ & $0.535 \pm 0.011$ & $2.40 \pm 0.15$ & $3^{a, d}$ \\
\hline 4914423 & $5.60 \pm 0.68$ & $1.117 \pm 0.059$ & $1.468 \pm 0.021$ & $0.3546 \pm 0.0058$ & $2.29 \pm 0.14$ & $2^{a}$ \\
\hline 4914923 & $6.37 \pm 0.33$ & $1.123 \pm 0.033$ & $1.388 \pm 0.014$ & $0.4207 \pm 0.0042$ & $2.00 \pm 0.11$ & \\
\hline 5094751 & $5.63 \pm 0.79$ & $1.072 \pm 0.041$ & $1.353 \pm 0.019$ & $0.4346 \pm 0.0098$ & $2.09 \pm 0.13$ & $2^{a}$ \\
\hline 5184732 & $4.84 \pm 0.35$ & $1.205 \pm 0.022$ & $1.3427 \pm 0.0085$ & $0.5034 \pm 0.0050$ & $1.912 \pm 0.098$ & \\
\hline 5773345 & $2.24 \pm 0.50$ & $1.510 \pm 0.044$ & $2.017 \pm 0.021$ & $0.1841 \pm 0.0026$ & $5.19 \pm 0.33$ & \\
\hline 5866724 & $2.53 \pm 0.42$ & $1.273 \pm 0.032$ & $1.423 \pm 0.017$ & $0.4434 \pm 0.0064$ & $2.648 \pm 0.098$ & $3^{e}$ \\
\hline 5950854 & $9.8 \pm 1.1$ & $0.984 \pm 0.044$ & $1.236 \pm 0.023$ & $0.524 \pm 0.012$ & $1.62 \pm 0.14$ & \\
\hline 6106415 & $4.98 \pm 0.50$ & $1.069 \pm 0.038$ & $1.219 \pm 0.018$ & $0.596 \pm 0.011$ & $1.78 \pm 0.12$ & \\
\hline 6116048 & $6.80 \pm 0.63$ & $1.002 \pm 0.031$ & $1.218 \pm 0.014$ & $0.5602 \pm 0.0080$ & $1.78 \pm 0.11$ & \\
\hline 6196457 & $4.5 \pm 1.0$ & $1.268 \pm 0.069$ & $1.756 \pm 0.029$ & $0.2359 \pm 0.0042$ & $3.29 \pm 0.25$ & $3^{f, g}$ \\
\hline $6225718^{13}$ & $3.02 \pm 0.51$ & $1.156 \pm 0.043$ & $1.234 \pm 0.016$ & $0.6230 \pm 0.0082$ & $2.19 \pm 0.13$ & \\
\hline 6278762 & $10.90 \pm 0.86$ & $0.753 \pm 0.012$ & $0.7588 \pm 0.0045$ & $1.741 \pm 0.023$ & $0.343 \pm 0.022$ & $5^{h}$ \\
\hline 6508366 & $2.49 \pm 0.19$ & $1.492 \pm 0.052$ & $2.177 \pm 0.032$ & $0.1446 \pm 0.0024$ & $6.91 \pm 0.47$ & \\
\hline 6521045 & $6.44 \pm 0.35$ & $1.091 \pm 0.031$ & $1.525 \pm 0.011$ & $0.3090 \pm 0.0034$ & $2.43 \pm 0.14$ & $3^{a}$ \\
\hline 6603624 & $7.77 \pm 0.45$ & $1.065 \pm 0.023$ & $1.173 \pm 0.012$ & $0.668 \pm 0.016$ & $1.283 \pm 0.059$ & \\
\hline 6679371 & $2.13 \pm 0.12$ & $1.570 \pm 0.030$ & $2.222 \pm 0.017$ & $0.1432 \pm 0.0018$ & $7.90 \pm 0.44$ & \\
\hline 6933899 & $6.61 \pm 0.28$ & $1.104 \pm 0.023$ & $1.599 \pm 0.012$ & $0.2712 \pm 0.0020$ & $2.67 \pm 0.18$ & \\
\hline 7103006 & $2.09 \pm 0.45$ & $1.482 \pm 0.056$ & $1.954 \pm 0.026$ & $0.1985 \pm 0.0038$ & $5.60 \pm 0.38$ & \\
\hline $7106245^{10}$ & $8.36 \pm 0.72$ & $0.851 \pm 0.025$ & $1.048 \pm 0.014$ & $0.752 \pm 0.017$ & $1.363 \pm 0.098$ & \\
\hline 7199397 & $3.63 \pm 0.33$ & $1.308 \pm 0.034$ & $2.526 \pm 0.028$ & $0.0821 \pm 0.0014$ & $6.69 \pm 0.29$ & \\
\hline 7206837 & $1.80 \pm 0.55$ & $1.400 \pm 0.041$ & $1.584 \pm 0.016$ & $0.3516 \pm 0.0052$ & $3.58 \pm 0.18$ & \\
\hline 7296438 & $6.60 \pm 0.40$ & $1.158 \pm 0.034$ & $1.405 \pm 0.017$ & $0.4187 \pm 0.0045$ & $2.00 \pm 0.11$ & \\
\hline $7510397^{4}$ & $3.97 \pm 0.35$ & $1.206 \pm 0.030$ & $1.784 \pm 0.015$ & $0.2127 \pm 0.0017$ & $4.18 \pm 0.28$ & \\
\hline 7670943 & $2.36 \pm 0.47$ & $1.309 \pm 0.046$ & $1.447 \pm 0.019$ & $0.4336 \pm 0.0055$ & $3.31 \pm 0.27$ & \\
\hline 7680114 & $7.57 \pm 0.85$ & $1.108 \pm 0.033$ & $1.411 \pm 0.022$ & $0.398 \pm 0.011$ & $2.07 \pm 0.14$ & \\
\hline 7771282 & $3.28 \pm 0.56$ & $1.353 \pm 0.064$ & $1.681 \pm 0.032$ & $0.2866 \pm 0.0049$ & $3.89 \pm 0.23$ & \\
\hline 7871531 & $9.34 \pm 0.58$ & $0.830 \pm 0.022$ & $0.870 \pm 0.011$ & $1.274 \pm 0.025$ & $0.632 \pm 0.040$ & \\
\hline 7940546 & $2.80 \pm 0.20$ & $1.317 \pm 0.026$ & $1.899 \pm 0.018$ & $0.1924 \pm 0.0025$ & $4.96 \pm 0.24$ & \\
\hline 7970740 & $10.67 \pm 0.36$ & $0.760 \pm 0.014$ & $0.7819 \pm 0.0055$ & $1.610 \pm 0.013$ & $0.444 \pm 0.029$ & \\
\hline 8006161 & $4.75 \pm 0.25$ & $1.004 \pm 0.020$ & $0.9353 \pm 0.0070$ & $1.233 \pm 0.011$ & $0.717 \pm 0.040$ & \\
\hline 8077137 & $4.48 \pm 0.62$ & $1.150 \pm 0.043$ & $1.638 \pm 0.027$ & $0.2626 \pm 0.0056$ & $3.37 \pm 0.25$ & $2^{f}$ \\
\hline 8150065 & $3.28 \pm 0.55$ & $1.196 \pm 0.066$ & $1.393 \pm 0.032$ & $0.445 \pm 0.011$ & $2.57 \pm 0.20$ & \\
\hline 8179536 & $1.84 \pm 0.37$ & $1.248 \pm 0.059$ & $1.353 \pm 0.027$ & $0.5039 \pm 0.0081$ & $2.68 \pm 0.20$ & \\
\hline 8228742 & $4.42 \pm 0.49$ & $1.221 \pm 0.069$ & $1.791 \pm 0.041$ & $0.2128 \pm 0.0030$ & $4.11 \pm 0.36$ & \\
\hline 8292840 & $2.79 \pm 0.54$ & $1.099 \pm 0.036$ & $1.321 \pm 0.015$ & $0.4789 \pm 0.0060$ & $2.40 \pm 0.16$ & $3^{i}$ \\
\hline 8349582 & $8.68 \pm 0.53$ & $1.144 \pm 0.015$ & $1.4520 \pm 0.0066$ & $0.3742 \pm 0.0072$ & $2.003 \pm 0.086$ & $1^{a}$ \\
\hline
\end{tabular}

Notes. Columns are: age, mass, radius, mean density, luminosity, and number of confirmed exoplanets. The labels next to some of the KIC numbers refer to the outliers highlighted in Figs. 10 and 8. The footnotes in the planets column refer to the publication that announced or confirmed their discovery.

References. ${ }^{(a)}$ Marcy et al. (2014). ${ }^{(b)}$ Howell et al. (2012). ${ }^{(c)}$ Haywood et al. (2018). ${ }^{(d)}$ Steffen et al. (2012). ${ }^{(e)}$ Chaplin et al. (2013). ${ }^{(f)}$ Xie $(2014)$. ${ }^{(g)}$ Van Eylen \& Albrecht (2015). ${ }^{(h)}$ Campante et al. (2015). ${ }^{(i)}$ Rowe et al. (2014). ${ }^{(j)}$ Hadden \& Lithwick (2014). ${ }^{(k)}$ Barclay et al. $(2013) .{ }^{(l)}$ Pál et al. (2008). ${ }^{(m)}$ Gilliland et al. (2013). ${ }^{(n)}$ Carter et al. (2012). ${ }^{(o)}$ Steffen et al. (2013). ${ }^{(p)}$ Borucki et al. (2010b). ${ }^{(q)}$ Fressin et al. (2011). ${ }^{(r)}$ Batalha et al. (2011). ${ }^{(s)}$ Cochran et al. (1997). 
Table A.1. continued.

\begin{tabular}{|c|c|c|c|c|c|c|}
\hline KIC & $\tau / \mathrm{Gyr}$ & $M / M_{\odot}$ & $R / R_{\odot}$ & $\rho / \rho_{\odot}$ & $L / L_{\odot}$ & Planets \\
\hline $8379927^{2}$ & $1.84 \pm 0.29$ & $1.105 \pm 0.049$ & $1.122 \pm 0.017$ & $0.790 \pm 0.012$ & $1.55 \pm 0.13$ & \\
\hline 8394589 & $4.20 \pm 0.39$ & $1.024 \pm 0.037$ & $1.153 \pm 0.023$ & $0.673 \pm 0.019$ & $1.70 \pm 0.11$ & \\
\hline 8424992 & $10.00 \pm 0.94$ & $0.893 \pm 0.038$ & $1.033 \pm 0.025$ & $0.819 \pm 0.027$ & $1.034 \pm 0.083$ & \\
\hline $8478994^{9}$ & $5.68 \pm 0.96$ & $0.799 \pm 0.017$ & $0.776 \pm 0.014$ & $1.732 \pm 0.063$ & $0.482 \pm 0.032$ & $4^{j, k}$ \\
\hline 8494142 & $2.09 \pm 0.32$ & $1.458 \pm 0.056$ & $1.893 \pm 0.025$ & $0.2149 \pm 0.0060$ & $4.64 \pm 0.33$ & $2^{f}$ \\
\hline $8554498^{5}$ & $3.49 \pm 0.41$ & $1.320 \pm 0.026$ & $1.840 \pm 0.017$ & $0.2126 \pm 0.0030$ & $3.86 \pm 0.17$ & \\
\hline 8684730 & $2.55 \pm 0.45$ & $1.475 \pm 0.039$ & $2.105 \pm 0.039$ & $0.163 \pm 0.011$ & $5.01 \pm 0.25$ & \\
\hline $8694723^{11}$ & $5.26 \pm 0.23$ & $1.070 \pm 0.023$ & $1.515 \pm 0.013$ & $0.3084 \pm 0.0039$ & $3.16 \pm 0.19$ & \\
\hline $8760414^{8}$ & $15.78 \pm 0.91$ & $0.761 \pm 0.011$ & $0.9755 \pm 0.0090$ & $0.828 \pm 0.015$ & $1.032 \pm 0.064$ & \\
\hline 8866102 & $1.81 \pm 0.27$ & $1.223 \pm 0.054$ & $1.357 \pm 0.022$ & $0.4902 \pm 0.0048$ & $2.66 \pm 0.16$ & \\
\hline 8938364 & $11.02 \pm 0.58$ & $0.995 \pm 0.023$ & $1.363 \pm 0.011$ & $0.3955 \pm 0.0046$ & $1.74 \pm 0.10$ & \\
\hline $9025370^{1}$ & $5.77 \pm 0.60$ & $0.921 \pm 0.035$ & $0.987 \pm 0.019$ & $0.965 \pm 0.019$ & $0.709 \pm 0.084$ & \\
\hline 9098294 & $9.42 \pm 0.85$ & $0.934 \pm 0.041$ & $1.112 \pm 0.027$ & $0.682 \pm 0.021$ & $1.301 \pm 0.081$ & \\
\hline 9139151 & $1.82 \pm 0.19$ & $1.205 \pm 0.044$ & $1.168 \pm 0.016$ & $0.7566 \pm 0.0097$ & $1.96 \pm 0.12$ & \\
\hline 9139163 & $2.09 \pm 0.28$ & $1.378 \pm 0.039$ & $1.557 \pm 0.017$ & $0.3653 \pm 0.0038$ & $3.70 \pm 0.22$ & \\
\hline $9206432^{12}$ & $1.67 \pm 0.42$ & $1.355 \pm 0.047$ & $1.505 \pm 0.021$ & $0.3982 \pm 0.0065$ & $3.76 \pm 0.21$ & \\
\hline 9353712 & $2.44 \pm 0.21$ & $1.465 \pm 0.054$ & $2.164 \pm 0.032$ & $0.1446 \pm 0.0019$ & $6.60 \pm 0.50$ & \\
\hline 9410862 & $7.34 \pm 0.75$ & $0.954 \pm 0.028$ & $1.139 \pm 0.017$ & $0.650 \pm 0.019$ & $1.580 \pm 0.099$ & \\
\hline 9414417 & $2.80 \pm 0.22$ & $1.319 \pm 0.041$ & $1.882 \pm 0.023$ & $0.1978 \pm 0.0026$ & $4.89 \pm 0.29$ & \\
\hline 9592705 & $2.21 \pm 0.19$ & $1.521 \pm 0.044$ & $2.118 \pm 0.026$ & $0.1610 \pm 0.0036$ & $5.94 \pm 0.37$ & \\
\hline 9812850 & $1.99 \pm 0.39$ & $1.417 \pm 0.057$ & $1.805 \pm 0.025$ & $0.2399 \pm 0.0067$ & $4.71 \pm 0.24$ & \\
\hline 9955598 & $6.69 \pm 0.48$ & $0.921 \pm 0.032$ & $0.893 \pm 0.012$ & $1.306 \pm 0.015$ & $0.634 \pm 0.042$ & $1^{a}$ \\
\hline $9965715^{6}$ & $3.88 \pm 0.49$ & $1.030 \pm 0.023$ & $1.244 \pm 0.011$ & $0.538 \pm 0.013$ & $1.78 \pm 0.17$ & \\
\hline $10068307^{7}$ & $3.98 \pm 0.34$ & $1.271 \pm 0.045$ & $2.024 \pm 0.023$ & $0.1546 \pm 0.0015$ & $5.24 \pm 0.37$ & \\
\hline 10079226 & $2.56 \pm 0.40$ & $1.153 \pm 0.050$ & $1.160 \pm 0.016$ & $0.744 \pm 0.013$ & $1.521 \pm 0.088$ & \\
\hline 10162436 & $3.29 \pm 0.42$ & $1.379 \pm 0.052$ & $2.019 \pm 0.026$ & $0.1678 \pm 0.0017$ & $5.25 \pm 0.31$ & \\
\hline $10454113^{3}$ & $5.07 \pm 0.91$ & $1.078 \pm 0.048$ & $1.202 \pm 0.022$ & $0.659 \pm 0.018$ & $1.94 \pm 0.13$ & \\
\hline 10514430 & $7.01 \pm 0.61$ & $1.042 \pm 0.029$ & $1.578 \pm 0.016$ & $0.2664 \pm 0.0034$ & $2.55 \pm 0.16$ & \\
\hline 10516096 & $6.66 \pm 0.47$ & $1.108 \pm 0.035$ & $1.422 \pm 0.017$ & $0.3861 \pm 0.0029$ & $2.30 \pm 0.17$ & \\
\hline 10586004 & $6.13 \pm 0.89$ & $1.154 \pm 0.041$ & $1.643 \pm 0.029$ & $0.2605 \pm 0.0056$ & $2.73 \pm 0.20$ & $2^{i}$ \\
\hline 10644253 & $1.28 \pm 0.25$ & $1.128 \pm 0.027$ & $1.1204 \pm 0.0085$ & $0.804 \pm 0.012$ & $1.525 \pm 0.060$ & \\
\hline 10666592 & $2.02 \pm 0.25$ & $1.556 \pm 0.046$ & $2.009 \pm 0.023$ & $0.1918 \pm 0.0027$ & $5.92 \pm 0.42$ & $1^{l}$ \\
\hline 10730618 & $4.4 \pm 1.1$ & $1.249 \pm 0.085$ & $1.716 \pm 0.053$ & $0.251 \pm 0.012$ & $3.81 \pm 0.52$ & \\
\hline 10963065 & $4.57 \pm 0.48$ & $1.065 \pm 0.043$ & $1.225 \pm 0.026$ & $0.584 \pm 0.017$ & $1.93 \pm 0.13$ & $1^{a}$ \\
\hline 11081729 & $1.66 \pm 0.84$ & $1.363 \pm 0.039$ & $1.444 \pm 0.018$ & $0.4526 \pm 0.0082$ & $3.46 \pm 0.20$ & \\
\hline 11133306 & $4.58 \pm 0.74$ & $1.081 \pm 0.040$ & $1.198 \pm 0.016$ & $0.634 \pm 0.015$ & $1.66 \pm 0.11$ & \\
\hline 11253226 & $1.34 \pm 0.50$ & $1.420 \pm 0.071$ & $1.599 \pm 0.026$ & $0.3468 \pm 0.0094$ & $4.53 \pm 0.29$ & \\
\hline 11295426 & $6.31 \pm 0.39$ & $1.099 \pm 0.031$ & $1.251 \pm 0.013$ & $0.5673 \pm 0.0080$ & $1.587 \pm 0.096$ & $3^{m}$ \\
\hline 11401755 & $6.94 \pm 0.78$ & $1.043 \pm 0.031$ & $1.601 \pm 0.026$ & $0.2552 \pm 0.0074$ & $2.91 \pm 0.21$ & $2^{n}$ \\
\hline 11772920 & $9.9 \pm 1.1$ & $0.823 \pm 0.040$ & $0.845 \pm 0.018$ & $1.373 \pm 0.024$ & $0.468 \pm 0.068$ & \\
\hline 11807274 & $2.01 \pm 0.28$ & $1.306 \pm 0.032$ & $1.597 \pm 0.013$ & $0.3223 \pm 0.0052$ & $3.51 \pm 0.17$ & $2^{o}$ \\
\hline 11853905 & $6.60 \pm 0.57$ & $1.163 \pm 0.046$ & $1.588 \pm 0.019$ & $0.2911 \pm 0.0042$ & $2.53 \pm 0.17$ & $1^{p}$ \\
\hline 11904151 & $11.65 \pm 0.93$ & $0.880 \pm 0.031$ & $1.041 \pm 0.016$ & $0.784 \pm 0.022$ & $0.995 \pm 0.054$ & $2^{q, r}$ \\
\hline 12009504 & $3.80 \pm 0.29$ & $1.197 \pm 0.044$ & $1.411 \pm 0.018$ & $0.4266 \pm 0.0054$ & $2.63 \pm 0.16$ & \\
\hline 12069127 & $2.31 \pm 0.18$ & $1.537 \pm 0.051$ & $2.297 \pm 0.030$ & $0.1267 \pm 0.0015$ & $7.48 \pm 0.44$ & \\
\hline 12069424 & $6.92 \pm 0.25$ & $1.056 \pm 0.019$ & $1.213 \pm 0.011$ & $0.5967 \pm 0.0085$ & $1.531 \pm 0.076$ & \\
\hline 12069449 & $7.08 \pm 0.22$ & $1.0000 \pm 0.0086$ & $1.101 \pm 0.012$ & $0.758 \pm 0.018$ & $1.201 \pm 0.024$ & $1^{s}$ \\
\hline 12258514 & $4.40 \pm 0.29$ & $1.252 \pm 0.054$ & $1.614 \pm 0.023$ & $0.2999 \pm 0.0024$ & $2.95 \pm 0.18$ & \\
\hline 12317678 & $2.63 \pm 0.28$ & $1.288 \pm 0.036$ & $1.775 \pm 0.022$ & $0.2301 \pm 0.0042$ & $5.33 \pm 0.25$ & \\
\hline
\end{tabular}

